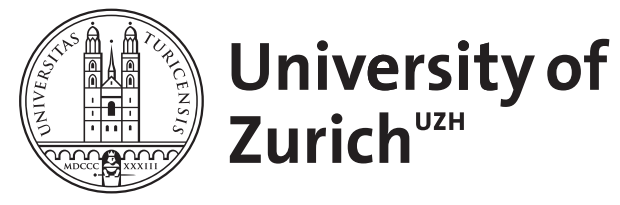

\title{
Nonautonomous parabolic equations involving measures
}

\author{
Amann, H
}

\begin{abstract}
In the first part of this paper, we study abstract parabolic evolution equations involving Banach space-valued measures. These results are applied in the second part to second-order parabolic systems under minimal regularity hypotheses on the coefficients.
\end{abstract}

DOI: https://doi.org/10.1007/s10958-005-0376-8

Posted at the Zurich Open Repository and Archive, University of Zurich ZORA URL: https://doi.org/10.5167/uzh-21838

Journal Article

Accepted Version

Originally published at:

Amann, H (2005). Nonautonomous parabolic equations involving measures. Journal of Mathematical Sciences (New York), 130(4):4780-4802.

DOI: https://doi.org/10.1007/s10958-005-0376-8 


\title{
NONAUTONOMOUS PARABOLIC EQUATIONS INVOLVING MEASURES
}

\author{
H. AMANN
}

\section{Dedicated to V.A. Solonnikov for his $70^{\text {th }}$ birthday}

Summary: In the first part of this paper we study abstract parabolic evolution equations involving Banach space valued measures. These results are applied in the second part to second order parabolic systems under minimal regularity hypotheses on the coefficients.

\section{INTRODUCTION}

In [5] we developed a solvability and regularity theory for abstract parabolic evolution equations of the form

$$
\dot{u}+A u=\mu \quad \text { in } J .
$$

Here $-A$ generates an analytic semigroup on some Banach space $E$, and $\mu$ is a bounded Radon measure on a bounded interval $J$, taking values in a suitable intermediate space between $E$ and the domain of $A$. In that paper it is also shown that the general theory applies to linear parabolic boundary value problems of the form

$$
\begin{aligned}
\partial_{t} u+\mathcal{A} u & =\mu_{\Omega} \quad \text { in } \Omega \times J, \\
\mathcal{B} u & =\mu_{\Gamma} \quad \text { on } \Gamma \times J,
\end{aligned}
$$

where $\Omega \subset \mathbb{R}^{n}$ and $\Gamma$ is the boundary of $\Omega$. In this case $\mu_{\Omega}$ is a bounded Radon measure on $\Omega \times J$ and $\mu_{\Gamma}$ is such a measure on $\Gamma \times J$.

In [10] the semilinear analogues of $(0.1)$ and $(0.2)$, where $\mu$ and $\left(\mu_{\Omega}, \mu_{\Gamma}\right)$, respectively, may also depend nonlinearly and nonlocally on the unknown solution $u$, are investigated. Based on the results of [5], general existence, uniqueness, and continuity theorems are proved and it is shown that they have numerous applications to semilinear parabolic problems (also see [9] for applications to control problems).

Formally, the solution of $(0.1)$ is given by $U \star \mu$, where $U$ is the semigroup generated by $-A$. The difficulty lies in defining a convolution of $U$ with a Banach space valued measure possessing suitable regularity properties which reflect the parabolicity of problem (0.1). In [5] this problem is resolved by a detailed study of semigroups in spaces of integrable functions, and a duality approach.

In [10, Section 16] it is shown how the results of [5] can be extended to the nonautonomous case. Nonautonomous equations arise naturally in linearization procedures. In this connection it is important that only minimal regularity conditions are required. (See [10, Section 19], where the 
case of certain parabolic equations with lower order coefficients possessing only weak regularity properties is treated in a somewhat ad hoc fashion.)

In this paper we present a different approach to problem (0.1) which works equally well in the autonomous and the nonautonomous case. In contrast to [5], where no further restriction on the generator $-A$ is needed, we now impose maximal $L_{p}$ regularity hypotheses. Given theses assumptions, the theory for the linear problem (0.1), again based on a duality argument, is now much easier and gives sharper results (even in the autonomous case) than the one of [5].

This paper consists of two parts. In the first one we study problem (0.1), given maximal regularity hypotheses. In particular, we give a natural definition of a solution to this problem if $\mu$ is a Banach space valued bounded Radon measure on $J$. The main result is the existence, uniqueness, and continuity theorem of Section 8. It extends and sharpens the theory of [5] for the case of a constant $A$, modulo the maximal regularity hypothesis, of course.

In the second one we show how the general results can be applied to nonautonomous parabolic systems involving general Radon measures, and even more singular distributions, in $\Omega \times J$ and on $\Gamma \times J$. The main result there is Theorem 16.1. Its strength lies in the fact that we require minimal regularity hypotheses for the coefficients of the differential operators (except for the $x$ dependence of the top order ones).

We close this introduction by two particular cases of Theorem 16.1 for which we choose a simple setting. Thus we assume that $\Omega$ is a bounded domain in $\mathbb{R}^{n}$ with a smooth boundary lying locally on one side of it. Moreover,

- $\boldsymbol{a} \in C\left([0, T], C^{1}\left(\bar{\Omega}, \mathbb{R}^{n \times n}\right)\right)$, and it is symmetric and uniformly positive definit.

Then we put

$$
\mathcal{A} u:=-\nabla \cdot(\boldsymbol{a} \nabla u)+a u .
$$

In order to specify the regularity assumptions for $a$ we suppose that

$$
q>1, \quad s \geq 0, \quad 0 \leq 2 / \rho+s<1-n / q^{\prime} .
$$

Note that this implies

$$
1<q<n /(n-1)
$$

We assume that

$$
\text { - } \quad a \in L_{\rho}\left((0, T), L_{\xi}(\Omega)\right) \text {, }
$$

where

$$
0 \leq 1 / \xi \leq s / n+1 / q^{\prime}
$$

We also suppose that

$$
\left(\nu_{0}, \vec{\nu}, \mu_{\Gamma}\right) \in \mathcal{M}(\Omega \times[0, T)) \times \mathcal{M}\left(\bar{\Omega} \times[0, T), \mathbb{R}^{n}\right) \times \mathcal{M}(\Gamma \times[0, T)),
$$

where $\mathcal{M}$ is the space of bounded Radon measures. Then we define a singular distribution on $\bar{\Omega}$ of order at most one by

$$
\mu_{\Omega}:=\nu_{0}+\nabla \cdot \vec{\nu} .
$$


First we consider the Dirichlet problem

$$
\begin{aligned}
\partial_{t} u+\mathcal{A} u=\mu_{\Omega} & \text { in } \Omega \times[0, T), \\
u=\mu_{\Gamma} & \text { on } \Gamma \times[0, T) .
\end{aligned}
$$

By an $L_{r}\left(W_{q}^{s}\right)$ solution of $(0.6)$ we mean a $u \in L_{r}\left((0, T), W_{q}^{s}(\Omega)\right)$ satisfying

$$
\begin{aligned}
\int_{0}^{T} & \int_{\Omega}(-\dot{\varphi}-\nabla \cdot(\boldsymbol{a} \nabla \varphi)+a \varphi) u d x d t \\
\quad & =\int_{\Omega \times[0, T)} \varphi d \nu_{0}-\int_{\bar{\Omega} \times[0, T)} \nabla \varphi \cdot d \vec{\nu}-\int_{\Gamma \times[0, T)} \partial_{\nu} \varphi d \mu_{\Gamma}
\end{aligned}
$$

for all $\varphi \in \mathcal{D}(\bar{\Omega} \times[0, T))$ with $\varphi(\cdot, t) \mid \Gamma=0$ for $0 \leq t<T$. Here $\partial_{\nu}$ is the outer conormal derivative with respect to $\boldsymbol{a}$, and $\mathcal{D}$ is the space of smooth functions with compact support (in the manifold indicated in the parentheses). Furthermore, in the first integral on the right-hand side the measure $\nu_{0}$ is identified with its unique extension in $\mathcal{M}(\bar{\Omega} \times[0, T))$ assigning the value zero to every Borel subset of $\Gamma \times[0, T)$. Observe that $(0.7)$ is formally obtained from (0.6) by multiplying the differential equation by $\varphi$ and using Green's formula, the boundary condition, and the (natural) definition of $\nabla \cdot \vec{\nu}$.

Besides of (0.6) we also consider the Neumann problem

$$
\begin{aligned}
\partial_{t} u+\mathcal{A} u=\mu_{\Omega} & \text { in } \Omega \times[0, T), \\
\partial_{\boldsymbol{\nu}} u=\mu_{\Gamma} & \text { on } \Gamma \times[0, T),
\end{aligned}
$$

where $\partial_{\boldsymbol{\nu}}$ is the conormal derivative with respect to $\boldsymbol{a}$. In this case, instead of (0.4), we suppose that

$$
\text { - } 0 \leq 1 / \xi \leq(1+s) / n+1 / q^{\prime}
$$

with $\xi \geq 1$, of course. Moreover, we replace (0.5) by

$$
\left(\mu_{\Omega}, \mu_{\Gamma}\right) \in \mathcal{M}(\Omega) \times \mathcal{M}(\Gamma) .
$$

An $L_{r}\left(W_{q}^{1+s}\right)$ solution of $(0.8)$ is a $u \in L_{r}\left((0, T), W_{q}^{1+s}(\Omega)\right)$ satisfying

$$
\begin{array}{r}
\int_{0}^{T} \int_{\Omega}(-\dot{\varphi} u+\nabla \varphi \cdot \boldsymbol{a} \nabla u+\varphi a u) d x d t \\
\quad=\int_{\Omega \times[0, T)} \varphi d \mu_{\Omega}+\int_{\Gamma \times[0, T)} \gamma \varphi d \mu_{\Gamma}
\end{array}
$$

for all $\varphi \in \mathcal{D}(\bar{\Omega} \times[0, T))$, where $\gamma$ denotes the trace operator. Clearly, $(0.10)$ is the natural weak form of $(0.8)$.

Theorem 0.1. Suppose that

$$
2-2 / \rho \geq 2 / r>1+s+n / q^{\prime}
$$

Then the Dirichlet problem (0.6) has a unique $L_{r}\left(W_{q}^{s}\right)$, and the Neumann problem (0.8) a unique $L_{r}\left(W_{q}^{1+s}\right)$, solution. In either case, it depends continuously on all data. If $\mu_{\Omega} \geq 0$ and $\mu_{\Gamma} \geq 0$ then $u \geq 0$.

Proof. This follows from Proposition 15.1, Theorems 16.1 and 17.1, and $[5$, Example 1(d)]. 
It should be remarked that the solution of the Dirichlet problem belongs also to $L_{r}\left((0, T), W_{q}^{1+s}(\Omega)\right)$, provided assumption $(0.9)$ is satisfied and $\mu_{\Gamma}=0$.

We refer to Theorem 16.1 for a precise formulation of the continuous parameter dependence and further continuity assertions. The latter theorem takes also care of lower order derivatives and applies to general systems and unbounded domains.

Theorem 0.1 describes a rather precise interplay between the regularity of the coefficient $a$ and the one of the solution. To illustrate this, we restrict ourselves to the Dirichlet problem, for simplicity.

In the following, given a real number $\alpha$, we put $\alpha+:=\beta$ [resp. $\alpha-:=\beta]$ to indicate that $\beta>\alpha$ [resp. $\beta<\alpha]$ and that $\beta$ is as close to $\alpha$ as we please.

First we suppose that $a$ has only little space regularity. It follows from (0.4), by setting $\rho=\infty$ and $s=\left(1-n / q^{\prime}\right)-$ in (0.3), that we must have $\xi=n+$ at least. No matter how we choose $s<1-n / q^{\prime}$, Sobolev's embedding theorem implies $W_{q}^{s}(\Omega) \hookrightarrow L_{r}(\Omega)$ with $r:=[n /(n-1)]-$. Furthermore, (0.11) requires $r=1+$. In summary:

- if $a \in L_{\infty}\left((0, T), L_{n+}(\Omega)\right)$, then (0.6) has a unique $L_{1+}\left(L_{[n /(n-1)]-}\right)$ solution.

Next we assume that $a$ has little time regularity. Due to $(0.3)$ we must have $\rho=2+$ at least, which occurs for $s=0$ and $q=1+$. Then we infer from (0.4) that (practically) $\xi=\infty$. Thus (0.11) implies that

- if $a \in L_{2+}\left((0, T), L_{\infty}(\Omega)\right)$, then (0.6) has a unique $L_{2-}\left(L_{1+}\right)$ solution.

Consequently,

- if $a \in L_{\infty}\left((0, T), L_{\infty}(\Omega)\right)$, then (0.6) has a unique solution $u \in L_{2-}\left((0, T), L_{1+}(\Omega)\right) \cap L_{1+}\left((0, T), L_{[n /(n-1)]-}(\Omega)\right)$.

Indeed, this follows from (0.11), that is, Theorem 0.1 , since, by uniqueness, an $L_{r}\left(W_{q}^{s}\right)$ solution is independent of $s, r, \rho$, and $q$ as long as $(0.3),(0.4)$ [resp, (0.9)], and (0.11) are satisfied.

Linear nonautonomous parabolic problems have already been discussed in [10, Theorem 19.4] in the particular situation where $\boldsymbol{a}$ is independent of $t$. The main improvement of Theorem 16.1 over the earlier result is the fact that $\boldsymbol{a}$ is time-dependent, where only continuity is required. This is achieved by employing maximal regularity.

For simplicity, we impose the rather strong $C^{1}$ space regularity assumption on $\boldsymbol{a}$. Weaker restrictions will be discussed elsewhere.

\section{Part 1. Abstract evolution equations involving measures}

In this part we study the abstract evolution equation (0.1) under maximal regularity assumptions. By a solution we mean a weak solution defined below in a natural way. The keys for our approach are embedding theorems and a duality argument. 
Given Banach spaces $E$ and $F$, we denote by $\mathcal{L}(E, F)$ the Banach space of all bounded linear operators from $E$ into $F$, and $\mathcal{L}(E):=\mathcal{L}(E, E)$. We write $\mathcal{L}$ is $(E, F)$ for the set of all isomorphisms in $\mathcal{L}(E, F)$.

We denote by $\mathcal{H}(F)$ the set of closed and densely defined linear operators $A$ in $F$ such that $-A$ generates a strongly continuous analytic semigroup, $\left\{e^{-t A} ; t \geq 0\right\}$, on $F$. If $E \stackrel{d}{\hookrightarrow} F$, that is, $E$ is continuously and densely embedded in $F$, then $\mathcal{H}(E, F):=\mathcal{L}(E, F) \cap \mathcal{H}(F)$.

Throughout this part

- $E_{0}$ and $E_{1}$ are Banach spaces with

$\left.\begin{array}{ll} & E_{1} \stackrel{d}{\hookrightarrow} E_{0} \text { and } \mathcal{H}\left(E_{1}, E_{0}\right) \neq \emptyset ; \\ \text { - } & 1<p<\infty .\end{array}\right\}$

\section{Preliminaries}

Let $E_{0}$ and $E_{1}$ be Banach spaces such that $E_{1} \stackrel{d}{\hookrightarrow} E_{0}$. We write $[\cdot, \cdot]_{\theta}$ for the complex and $(\cdot, \cdot)_{\theta, r}, 1 \leq r \leq \infty$, for the real interpolation functors of exponent $\theta \in(0,1)$ (see [4, Section I.2], for example, for a summary of interpolation theory, and [11] or [16] for proofs). Then $E_{[\theta]}:=\left[E_{0}, E_{1}\right]_{\theta}$ and $E_{\theta, r}:=\left(E_{0}, E_{1}\right)_{\theta, r}$ for $1 \leq r \leq \infty$ and $0<\theta<1$. Recall that

$$
E_{1} \stackrel{d}{\hookrightarrow} E_{\theta, 1} \stackrel{d}{\hookrightarrow} E_{\theta, q} \stackrel{d}{\hookrightarrow} E_{\theta, p} \hookrightarrow E_{\theta, \infty} \stackrel{d}{\hookrightarrow} E_{\vartheta, 1} \stackrel{d}{\hookrightarrow} E_{0}
$$

for $1<q<p<\infty$ and $0<\vartheta<\theta<1$. Moreover,

$$
E_{\theta, 1} \stackrel{d}{\hookrightarrow} E_{[\theta]} \hookrightarrow E_{\theta, \infty}, \quad 0<\theta<1 .
$$

For convenience, we set $E_{j, r}:=E_{j}$ for $j=0,1$ and $1 \leq r \leq \infty$.

Let $J$ be a subinterval of $\mathbb{R}^{+}$containing 0 such that $\overline{\dot{J}}:=J \backslash\{0\} \neq \emptyset$. Given $1 \leq p<\infty$, put

$$
\mathbb{W}_{p}^{1}(J):=\mathbb{W}_{p}^{1}\left(J,\left(E_{1}, E_{0}\right)\right):=W_{p}^{1}\left(J, E_{0}\right) \cap L_{p}\left(J, E_{1}\right) .
$$

Then

$$
\mathbb{W}_{p}^{1}\left(J,\left(E_{1}, E_{0}\right)\right) \hookrightarrow\left\{\begin{array}{cl}
L_{q}\left(J, E_{\theta, 1}\right) & \text { if } 1 \geq 1 / q>\theta-1 / p^{\prime}>0, \\
C\left(J, E_{1 / p^{\prime}, p}\right) & \text { if } \theta=1 / p^{\prime}, \\
C^{\rho}\left(J, E_{\theta, 1}\right) & \text { if } 0 \leq \rho<1 / p^{\prime}-\theta .
\end{array}\right.
$$

Moreover, if $\theta \neq 1 / p^{\prime}$ and $E_{1}$ is compactly embedded in $E_{0}$ then these embeddings are compact as well (see [5, Theorem 3] and Theorem III.4.10.2 of [4]). Hence, given $\tau \in J$, the trace map, $\gamma_{\tau}$, is well-defined by $u \mapsto u(\tau)$, and

$$
\gamma_{\tau} \in \mathcal{L}\left(\mathbb{W}_{p}^{1}(J), E_{1 / p^{\prime}, p}\right)
$$

We set

$$
\mathbb{W}_{p, \tau}^{1}(J):=\mathbb{W}_{p, \tau}^{1}\left(J,\left(E_{1}, E_{0}\right)\right):=\left\{u \in \mathbb{W}_{p}^{1}(J) ; \gamma_{\tau} u=0\right\}, \quad \tau \in J .
$$




\section{Definitions AND ELEMENTARY PROPERTIES}

For $T>0$ we put $J_{T}:=[0, T]$. Then we

- $\quad$ fix $\mathrm{T}>0$ and set $\mathrm{J}:=J_{\mathrm{T}}$.

Henceforth, given a map $A: \mathrm{J} \rightarrow \mathcal{L}\left(E_{1}, E_{0}\right)$, we identify it with its pointwise extension, defined by $(A u)(t):=A(t) u(t)$ for $t \in \mathrm{J}$ and $u: J \rightarrow E_{1}$. The relation

$$
A \in L_{1}\left(\mathrm{~J}, \mathcal{L}\left(E_{1}, E_{0}\right)\right) \cap \mathcal{L}\left(\mathbb{W}_{p}^{1}\left(\mathrm{~J},\left(E_{1}, E_{0}\right)\right), L_{p}\left(\mathrm{~J}, E_{0}\right)\right)
$$

is always to be understood in this sense.

Let (2.1) be satisfied and consider the Cauchy problem

$$
\dot{u}+A u=f \quad \text { in } \mathbf{j}, \quad u(0)=x,
$$

where

$$
(f, x) \in L_{p}\left(\mathrm{~J}, E_{0}\right) \times E_{1 / p^{\prime}, p} .
$$

By a (strong) $L_{p}\left(E_{1}\right)$ solution of $(2.2)$ (on $\mathrm{J}$ ) we mean a function $u \in \mathbb{W}_{p}^{1}(\mathrm{~J})$ satisfying (2.2) (in the point-wise sense a.e. or, equivalently, in the distributional sense). Note that, thanks to (1.2), the initial condition has a well-defined meaning.

The operator $A$, satisfying hypothesis (2.1), is said to possess the property of maximal $L_{p}$ regularity (on $\mathrm{J}$ with respect to $\left(E_{1}, E_{0}\right)$ ) if one, hence all, of the conditions of the following proposition are satisfied.

Proposition 2.1. Let (2.1) be satisfied. Then the following are equivalent:

(i) $\partial+A \in \mathcal{L}$ is $\left(\mathbb{W}_{p, 0}^{1}\left(\mathrm{~J},\left(E_{1}, E_{0}\right)\right), L_{p}\left(\mathrm{~J}, E_{0}\right)\right)$.

(ii) The Cauchy problem (2.2) has for $x=0$ and each $f \in L_{p}\left(\mathrm{~J}, E_{0}\right)$ a unique $L_{p}\left(E_{1}\right)$ solution.

(iii) $\left(\partial+A, \gamma_{0}\right) \in \mathcal{L}$ is $\left(\mathbb{W}_{p}^{1}(\mathrm{~J}), L_{p}\left(\mathrm{~J}, E_{0}\right) \times E_{1 / p^{\prime}, p}\right)$.

(iv) Problem (2.2) has for each $(f, x)$ satisfying $(2.3)$ a unique $L_{p}\left(E_{1}\right)$ solution.

Proof. The map $\partial+A$ [resp. $\left.\left(\partial+A, \gamma_{0}\right)\right]$ is linear and continuous from $\mathbb{W}_{p, 0}^{1}(\mathrm{~J})\left[\operatorname{resp} . \mathbb{W}_{p}^{1}(\mathrm{~J})\right]$ into $L_{p}\left(\mathrm{~J}, E_{0}\right)\left[\operatorname{resp} . L_{p}\left(\mathrm{~J}, E_{0}\right) \times E_{1 / p^{\prime}, p}\right]$. It is bijective iff (ii) [resp. (iv)] is true. Hence the equivalence of (i) and (ii) [resp. (iii) and (iv)] follows from the open mapping theorem. Clearly, (iv) implies (ii). Given $(f, x)$ satisfying $(2.3)$, set $v(t):=e^{-t C} x$ for $t \in \mathrm{J}$ and some $C \in \mathcal{H}\left(E_{1}, E_{0}\right)$. Then $v \in \mathbb{W}_{p}^{1}\left(\mathrm{~J},\left(E_{1}, E_{0}\right)\right.$ ) (cf. [4, Proposition III.4.10.2]). Thus $u \in \mathbb{W}_{p}^{1}(\mathrm{~J})$ satisfies $(2.2)$ iff $u=v+w$, where $w \in \mathbb{W}_{p}^{1}(\mathrm{~J})$ solves

$$
(\partial+A) w=f-(\partial+A) v \quad \text { in } \mathbf{j}, \quad w(0)=0 .
$$

This proves that (ii) implies (iv).

We denote by

$$
\mathcal{M R}_{p}(\mathrm{~J}):=\mathcal{M R}_{p}\left(\mathrm{~J},\left(E_{1}, E_{0}\right)\right)
$$

the set of all $A$ satisfying (2.1) and having the property of maximal $L_{p}$ regularity on $\mathrm{J}$ with respect to $\left(E_{1}, E_{0}\right)$.

Given $A \in \mathcal{M R}_{p}(\mathrm{~J})$, we put

$$
K:=K_{A}:=\left(\partial+A, \gamma_{0}\right)^{-1} \mid\left(L_{p}\left(\mathrm{~J}, E_{0}\right) \times\{0\}\right)
$$


and

$$
U:=U_{A}:=\left(\partial+A, \gamma_{0}\right)^{-1} \mid\left(\{0\} \times E_{1 / p^{\prime}, p}\right) .
$$

Using obvious identifications, it follows that

$$
K \in \mathcal{L} \operatorname{is}\left(L_{p}\left(\mathrm{~J}, E_{0}\right), \mathbb{W}_{p, 0}^{1}(\mathrm{~J})\right), \quad U \in \mathcal{L}\left(E_{1 / p^{\prime}, p}, \mathbb{W}_{p}^{1}(\mathrm{~J})\right) .
$$

Clearly, $u$ is the $L_{p}\left(E_{1}\right)$ solution of (2.2) iff $u=K f+U x$.

\section{CRIteria FOR MAXIMAL REgUlarity}

We denote by $\mathcal{C}(E)$ the set of all closed linear operators in the Banach space $E$. Then we set

$$
\mathcal{M R}_{p}\left(E_{1}, E_{0}\right):=\mathcal{L}\left(E_{1}, E_{0}\right) \cap \mathcal{C}\left(E_{0}\right) \cap \mathcal{M R}_{p}\left(\mathrm{~J},\left(E_{1}, E_{0}\right)\right)
$$

where we identify $A \in \mathcal{L}\left(E_{1}, E_{0}\right)$ with the constant map $t \mapsto A$.

Remarks 3.1. (a) The assumption that $A \in \mathcal{L}\left(E_{1}, E_{0}\right) \cap \mathcal{C}\left(E_{0}\right)$ is equivalent to $A \in \mathcal{L}\left(E_{1}, E_{0}\right)$ and $E_{1} \doteq D(A)$, where $D(A)$ is the domain of $A$ (in $E_{0}$ ) endowed with its graph norm.

Proof. See Lemma I.1.1.2 in [4].

(b) $\mathcal{M R}_{p}\left(E_{1}, E_{0}\right) \subset \mathcal{H}\left(E_{1}, E_{0}\right)$.

Proof. This is a result of Dore [14] (see [4, Remark III.4.10.9(b)]).

(c) For $A \in \mathcal{H}\left(E_{1}, E_{0}\right)$ put $V(t):=e^{-t A}$. Also set

$$
V \star f(t):=\int_{0}^{t} V(t-\tau) f(\tau) d \tau, \quad f \in L_{1}\left(\mathrm{~J}, E_{0}\right), \quad t \in \mathrm{J} .
$$

Then, if $A \in \mathcal{M R}_{p}\left(E_{1}, E_{0}\right)$, it follows that $K f=V \star f$ and $U x=V(\cdot) x$ for $(f, x)$ satisfying $(2.3)$.

Proof. This is a consequence of [4, Theorem III.1.5.2].

(d) If $A \in \mathcal{M R}_{p}\left(E_{1}, E_{0}\right)$ then $A \in \mathcal{M R}_{q}\left(E_{1}, E_{0}\right)$ for $1<q<\infty$.

Proof. This is implied by (a) and [15] (also see [14]).

We close this section with the following perturbation theorem for maximal regularity.

Theorem 3.2. If $\vartheta, \theta \in(0,1)$ and $0 \leq 1 / \rho<(1-\theta) \wedge 1 / p$ then

$$
C\left(\mathrm{~J}, \mathcal{M R}_{p}\left(E_{1}, E_{0}\right)\right)+L_{\infty}\left(\mathrm{J}, \mathcal{L}\left(E_{1}, E_{\vartheta, \infty}\right)\right)+L_{\rho}\left(\mathrm{J}, \mathcal{L}\left(E_{\theta, \infty}, E_{0}\right)\right)
$$

is a subset of $\mathcal{M R}_{p}\left(\mathrm{~J},\left(E_{1}, E_{0}\right)\right)$.

Proof. This is proved in [6]. 


\section{Estimates}

In this section we derive simple but important estimates for the solution of (2.2) if $f$ has better integrability properties.

Proposition 4.1. Suppose that $A \in \mathcal{M R}_{p}(\mathrm{~J})$ and $p \leq r \leq \infty$. Then

$$
\|K f\|_{\mathbb{W}_{p}^{1}\left(J_{T}\right)} \leq c T^{1 / p-1 / r}\|f\|_{L_{r}\left(J_{T}\right)}
$$

for $T \in \dot{\mathrm{j}}$ and $f \in L_{r}\left(J_{T}, E_{0}\right)$.

Proof. This follows from Hölder's inequality.

Theorem 4.2. Suppose that $A \in \mathcal{M R}_{p}(\mathrm{~J})$ and $p \leq r \leq \infty$. Then

$$
K \in \begin{cases}\mathcal{L}\left(L_{r}\left(J_{T}, E_{0}\right), L_{q}\left(J_{T}, E_{\theta, 1}\right)\right), & 1 \geq 1 / q>\theta-1 / p^{\prime}>0, \\ \mathcal{L}\left(L_{r}\left(J_{T}, E_{0}\right), C\left(J_{T}, E_{\theta, p}\right)\right), & \theta=1 / p^{\prime}, \\ \mathcal{L}\left(L_{r}\left(J_{T}, E_{0}\right), C^{\rho}\left(J_{T}, E_{\theta, 1}\right)\right), & 0 \leq \rho<1 / p^{\prime}-\theta,\end{cases}
$$

for $T \in \mathrm{J}$. Moreover, the norms of these linear maps are bounded by $c T^{\varepsilon}$, where $\varepsilon:=1 / p-1 / r$. If $E_{1}$ embeds compactly in $E_{0}$ then $K$ is compact whenever $\theta \neq 1 / p^{\prime}$.

Proof. For $f \in L_{r}\left(J_{T}, E_{0}\right)$ denote by $f^{0}$ its extension by zero over $\mathrm{J}$. Then

$$
\left\|f^{0}\right\|_{L_{p}\left(\mathrm{~J}, E_{0}\right)} \leq T^{\varepsilon}\|f\|_{L_{r}\left(J_{T}, E_{0}\right)}, \quad T \in \mathbf{J} .
$$

Hence Proposition 2.1 implies

$$
\left\|K f^{0}\right\|_{\mathbb{W}_{p}^{1}(\mathrm{~J})} \leq c T^{\varepsilon}\|f\|_{L_{r}\left(J_{T}, E_{0}\right)}, \quad T \in \mathbf{j} .
$$

The assertion follows now by applying (1.2) and taking the restriction to $J_{T}$.

It should be noted that Theorem 4.2 is closely related to the estimates for the autonomous case derived in [5, Section 3].

\section{Green's Formula}

Now we suppose that

- $E_{0}$ is reflexive.

Since $E_{1}$ is isomorphic to $E_{0}$, thanks to $\mathcal{H}\left(E_{1}, E_{0}\right) \neq \emptyset, E_{1}$ is reflexive as well. Consequently,

$$
E_{0}^{\prime} \stackrel{d}{\hookrightarrow} E_{1}^{\prime} .
$$

Suppose that $A$ satisfies (2.1) and put $A^{\prime}(t):=A(t)^{\prime} \in \mathcal{L}\left(E_{0}^{\prime}, E_{1}^{\prime}\right)$ for a.a. $t \in \mathrm{J}$.

Proposition 5.1. If $u \in \mathbb{W}_{p}^{1}\left(\mathrm{~J},\left(E_{1}, E_{0}\right)\right)$ and $v \in \mathbb{W}_{p^{\prime}}^{1}\left(\mathrm{~J},\left(E_{0}^{\prime}, E_{1}^{\prime}\right)\right)$ then Green's formula,

$$
\begin{aligned}
& \int_{s}^{t}\langle v,(\partial+A) u\rangle_{E_{0}} d t+\langle v(s), u(s)\rangle_{E_{1 / p^{\prime}, p}} \\
& \quad=\int_{s}^{t}\left\langle\left(-\partial+A^{\prime}\right) v, u\right\rangle_{E_{1}} d t+\langle v(t), u(t)\rangle_{E_{1 / p^{\prime}, p}},
\end{aligned}
$$

holds for $0 \leq s<t \leq T$. 
Proof. First note that

$$
\left(E_{1 / p^{\prime}, p}\right)^{\prime}=\left(\left(E_{0}, E_{1}\right)_{1 / p^{\prime}, p}\right)^{\prime}=\left(E_{0}^{\prime}, E_{1}^{\prime}\right)_{1 / p^{\prime}, p^{\prime}}=\left(E_{1}^{\prime}, E_{0}^{\prime}\right)_{1 / p, p^{\prime}}
$$

by the duality properties of the real interpolation functors. Hence we infer from (1.3) that $\langle v(t), u(t)\rangle_{E_{1 / p^{\prime}, p}}$ is well-defined for $t \in \mathrm{J}$. Now the assertion is an easy consequence of [4, Proposition V.2.4.7] (also see [4, Proposition V.2.6.1]).

Remark 5.2. From [4, Proposition V.2.4.7] we infer that Green's formula remains valid for the limiting cases $p=1$ and $p=\infty$, provided the duality pairing $\langle\cdot, \cdot\rangle_{E_{1 / p^{\prime}, p}}$ is replaced by $\langle\cdot, \cdot\rangle_{E_{0}}$.

\section{WEAK AND STRONG SOLUTIONS}

Henceforth, we put $E_{0}^{\bullet}:=E_{1}^{\prime}, E_{1}^{\bullet}:=E_{0}^{\prime}$, and

$$
A^{\bullet}:=\left(t \mapsto A^{\prime}(\mathrm{T}-t)\right) \in L_{1}\left(\mathrm{~J}, \mathcal{L}\left(E_{1}^{\bullet}, E_{0}^{\bullet}\right)\right) .
$$

We suppose that

- $E_{0}$ is reflexive;

- $A \in \mathcal{M R}_{p}\left(\mathrm{~J},\left(E_{1}, E_{0}\right)\right)$;

- $\left.A^{\bullet} \in \mathcal{M R}_{p^{\prime}}\left(\mathrm{J},\left(E_{1}^{\bullet}, E_{0}^{\bullet}\right)\right).\right\}$

A simple change of variables shows, thanks to Proposition 2.1, that the last hypothesis is equivalent to

$$
\left(-\partial+A^{\prime}, \gamma_{\mathbf{T}}\right) \in \mathcal{L} \text { is }\left(\mathbb{W}_{p^{\prime}}^{1}\left(\mathrm{~J},\left(E_{1}^{\bullet}, E_{0}^{\bullet}\right)\right), L_{p^{\prime}}\left(\mathrm{J}, E_{0}^{\bullet}\right) \times E_{1 / p, p^{\prime}}^{\bullet}\right) .
$$

Thus

$$
K^{\bullet}:=\left(-\partial+A^{\prime}, \gamma_{\mathrm{T}}\right)^{-1} \mid\left(L_{p^{\prime}}\left(\mathrm{J}, E_{0}^{\bullet}\right) \times\{0\}\right)
$$

is well-defined, and, using obvious identifications,

$$
K^{\bullet} \in \mathcal{L} \operatorname{is}\left(L_{p^{\prime}}\left(\mathrm{J}, E_{0}^{\bullet}\right), \mathbb{W}_{p^{\prime}, \mathrm{T}}^{1}\left(\mathrm{~J},\left(E_{1}^{\bullet}, E_{0}^{\bullet}\right)\right)\right) .
$$

Suppose that

$$
(x, f) \in E_{1 / p^{\prime}, p} \times L_{p}\left(\mathrm{~J}, E_{0}\right) .
$$

Then $u$ is said to be a weak $L_{p}\left(E_{1}\right)$ solution of $(2.2)$ iff $u \in L_{p}\left(J, E_{1}\right)$ and

$$
\int_{0}^{T}\left\langle\left(-\partial+A^{\prime}\right) \varphi, u\right\rangle_{E_{1}} d t=\int_{0}^{T}\langle\varphi, f\rangle_{E_{0}} d t+\langle\varphi(0), x\rangle_{E_{1 / p^{\prime}, p}}
$$

for $\varphi \in \mathcal{D}\left([0, T), E_{1}^{\bullet}\right)$.

Proposition 6.1. Let (6.1) and (6.4) be true. Then $u$ is a weak $L_{p}\left(E_{1}\right)$ solution of (2.2) iff it is a strong $L_{p}\left(E_{1}\right)$ solution.

Proof. If $u$ is a strong $L_{p}\left(E_{1}\right)$ solution, then we see from Green's formula that it is a weak $L_{p}\left(E_{1}\right)$ solution. 
Conversely, suppose that $u \in L_{p}\left(\mathrm{~J}, E_{1}\right)$ satisfies (6.5). Since $A$ belongs to $\mathcal{M R}_{p}\left(\mathrm{~J},\left(E_{1}, E_{0}\right)\right)$, the function $v:=\left(\partial+A, \gamma_{0}\right)^{-1}(f, x)$ is a welldefined element of $\mathbb{W}_{p}^{1}\left(J,\left(E_{1}, E_{0}\right)\right)$. Hence Green's formula implies

$$
\begin{aligned}
\int_{0}^{T}\langle\varphi, f\rangle_{E_{0}} d t & =\int_{0}^{T}\langle\varphi,(\partial+A) v\rangle_{E_{0}} d t \\
& =\int_{0}^{T}\left\langle\left(-\partial+A^{\prime}\right) \varphi, v\right\rangle_{E_{1}} d t-\langle\varphi(0), x\rangle_{E_{1 / p^{\prime}, p}}
\end{aligned}
$$

for $\varphi \in \mathcal{D}\left([0, T), E_{1}^{\bullet}\right)$. Thus we infer from (6.5) that

$$
\int_{0}^{T}\left\langle\left(-\partial+A^{\prime}\right) \varphi, u-v\right\rangle_{E_{1}} d t=0
$$

for all $\varphi \in \mathcal{D}\left([0, T), E_{1}^{\bullet}\right)$. It is not difficult to see that $\mathcal{D}\left([0, T), E_{1}^{\bullet}\right)$ is dense in $\mathbb{W}_{p^{\prime}, \mathrm{T}}^{1}\left(\mathrm{~J},\left(E_{1}^{\bullet}, E_{0}^{\bullet}\right)\right)$. Hence (6.6) holds for all $\varphi$ in the latter space. This implies, thanks to (6.2), that

$$
\langle g, u-v\rangle_{L_{p}\left(\mathrm{~J}, E_{1}\right)}=0, \quad g \in L_{p^{\prime}}\left(\mathrm{J}, E_{0}^{\bullet}\right) .
$$

Hence $u=v$, that is, $u$ is a strong $L_{p}\left(E_{1}\right)$ solution.

Corollary 6.2. If (6.1) and (6.4) hold then problem (2.2) possesses exactly one weak $L_{p}\left(E_{1}\right)$ solution, namely the unique strong one.

\section{Evolution EQUATIONS With MEASURES}

Let $X$ be a $\sigma$-compact metric space and $E$ a Banach space. We refer to [5] for precise definitions of $E$-valued measures and denote by $\mathcal{M}(X, E)$ the Banach space of all $E$-valued bounded Radon measures on $X$.

As usual, $C_{0}(X, E)$ is the Banach space of all $E$-valued continuous functions on $X$ vanishing at infinity, endowed with the maximum norm. Then the (generalized) Riesz representation theorem guarantees that

$$
C_{0}(X, E)^{\prime}=\mathcal{M}\left(X, E^{\prime}\right)
$$

with respect to the duality pairing

$$
\langle\mu, \varphi\rangle_{C_{0}(X, E)}:=\int_{X} \varphi d \mu, \quad(\mu, \varphi) \in \mathcal{M}\left(X, E^{\prime}\right) \times C_{0}(X, E) .
$$

Let $\sigma$ be a positive Radon measure on $X$. We identify $f \in L_{1}\left(X, \sigma, E^{\prime}\right)$ with that measure in $\mathcal{M}\left(X, E^{\prime}\right)$ which is absolutely continuous with respect to $\sigma$ and has $f$ as density, that is, with $f d \sigma$. Then

$$
L_{1}\left(X, \sigma, E^{\prime}\right) \hookrightarrow \mathcal{M}\left(X, E^{\prime}\right) .
$$

In fact, it follows easily from (7.1) that $L_{1}\left(X, \sigma, E^{\prime}\right)$ is a closed linear subspace of $\mathcal{M}\left(X, E^{\prime}\right)$.

Let assumption (6.1) be satisfied. From (6.3) and (an obvious modification of the proof of) Theorem 4.2 we deduce that

$$
K^{\bullet} \in \mathcal{L}\left(L_{r^{\prime}}\left(J_{T}, E_{0}^{\bullet}\right), C_{0}\left([0, T), E_{1 / p, p^{\prime}}^{\bullet}\right)\right), \quad p^{\prime} \leq r^{\prime}<\infty, \quad 0<T \leq \mathrm{T},
$$


and that the norm of this linear operator is bounded by $c T^{1 / p^{\prime}-1 / r^{\prime}}$. Thus (7.1) and (5.1) imply

$$
\left(K^{\bullet}\right)^{\prime} \in \mathcal{L}\left(\mathcal{M}\left([0, T), E_{1 / p^{\prime}, p}\right), L_{r}\left(J_{T}, E_{1}\right)\right)
$$

and

$$
\left\|\left(K^{\bullet}\right)^{\prime}\right\| \leq c T^{1 / r-1 / p}
$$

for $1<r \leq p$ and $0<T \leq \mathrm{T}$.

Note that, given $\mu \in \mathcal{M}\left([0, T), E_{1 / p^{\prime}, p}\right)$, writing $u:=\left(K^{\bullet}\right)^{\prime} \mu$ is equivalent to

$$
\langle g, u\rangle_{L_{p}\left(\mathrm{~J}, E_{1}\right)}=\left\langle\mu, K^{\bullet} g\right\rangle_{C_{0}\left([0, \mathrm{~T}), E_{1 / p, p^{\prime}}\right)}, \quad g \in L_{p^{\prime}}\left(\mathrm{J}, E_{0}^{\bullet}\right) .
$$

Set $\varphi:=K^{\bullet} g$ and recall that $\varphi \in \mathbb{W}_{r^{\prime}, \mathrm{T}}^{1}\left(\mathrm{~J},\left(E_{0}^{\bullet}, E_{1}^{\bullet}\right)\right)$. It follows that (7.6) is equivalent to

$$
\int_{0}^{\top}\left\langle\left(-\partial+A^{\prime}\right) \varphi, u\right\rangle_{E_{1}} d t=\int_{[0, \mathrm{~T})} \varphi d \mu, \quad \varphi \in \mathcal{D}\left([0, \mathrm{~T}), E_{1}^{\bullet}\right),
$$

where we used the density of $\mathcal{D}\left([0, \mathrm{~T}), E_{1}^{\bullet}\right)$ in $\mathbb{W}_{r^{\prime}, \mathrm{T}}^{1}\left(J,\left(E_{1}^{\bullet}, E_{0}^{\bullet}\right)\right)$. By comparing (7.7) with (6.5) it is natural to call $u$ weak $L_{p}\left(E_{1}\right)$ solution of the parabolic evolution equation

$$
\dot{u}+A u=\mu \quad \text { in }[0, T)
$$

iff $u$ belongs to $L_{p}\left(\mathrm{~J}, E_{1}\right)$ and satisfies (7.7). Observe that we do not prescribe an initial value since such a condition is implicitly contained in $\mu$. In fact, if $\mu$ equals $x \delta_{0}+\mu_{0}$ with $\mu_{0}(\{0\})=0$ then the right-hand side of (7.7) takes the form

$$
\int_{(0, T)} \varphi d \mu_{0}+\langle\varphi(0), x\rangle_{E_{1 / p^{\prime}, p}} .
$$

Also note that this definition is the natural extension of the corresponding concept studied in Section 6.

Example 7.1. (Autonomous operators) Let (6.1) be satisfied an suppose that $A$ is independent of $t \in \mathrm{J}$. Then we write $A \in \mathcal{M R}_{p}\left(E_{1}, E_{0}\right)$. Thus $A^{\prime} \in \mathcal{M R}_{p^{\prime}}\left(E_{1}^{\bullet}, E_{0}^{\bullet}\right)$. Put $V(t):=e^{-t A}$ and

$$
W \circledast g(t):=\int_{t}^{\top} e^{-(\tau-t) A^{\prime}} g(\tau) d \tau, \quad g \in L_{1}\left(\mathrm{~J}, E_{0}^{\bullet}\right) .
$$

It follows from Remark 3.1(c) by an obvious change of variables that $K^{\bullet}=W \circledast$. Moreover (cf. the proof of [5, Lemma 6]),

$$
\left(K^{\bullet}\right)^{\prime} f=V \star f, \quad f \in L_{1}\left(\mathrm{~J}, E_{0}\right) .
$$

For this reason we set

$$
V \star \mu:=\left(K^{\bullet}\right)^{\prime} \mu, \quad \mu \in \mathcal{M}\left(\mathrm{J}, E_{1 / p^{\prime}, p}\right),
$$

thus defining (formally) a convolution of a strongly continuous semigroup and a Banach space valued measure. 


\section{THE MAIN THEOREM}

Let assumptions $(\mathrm{H})$ and $(6.1)$ be satisfied. Als suppose that

- $1 \leq r<p$

- $\left.B \in L_{1}\left(\mathrm{~J}, \mathcal{L}\left(E_{1}, E_{0}\right)\right) \cap \mathcal{L}\left(L_{r}\left(\mathrm{~J}, E_{1}\right), L_{1}\left(\mathrm{~J}, E_{1 / p^{\prime}, p}\right)\right).\right\}$

Then, given $\mu \in \mathcal{M}\left([0, \mathrm{~T}), E_{1 / p^{\prime}, p}\right)$, we consider the perturbed problem

$$
\dot{u}+(A+B) u=\mu \quad \text { in }[0, \mathrm{~T}) .
$$

Note that $A+B$ is no longer required to possess the property of maximal $L_{p}$ regularity. Clearly, by a weak $L_{r}\left(E_{1}\right)$ solution of $(8.2)$ we mean a $u \in L_{r}\left(\mathrm{~J}, E_{1}\right)$ satisfying

$$
\left.\int_{0}^{\top}\left\{\left\langle\left(-\partial+A^{\prime}\right) \varphi, u\right\rangle_{E_{1}}+\langle\varphi, B u\rangle_{E_{1 / p^{\prime}, p}}\right\rangle\right\} d t=\int_{[0, \mathrm{~T})} \varphi d \mu
$$

for all $\varphi \in \mathcal{D}\left([0, \mathrm{~T}), E_{1}^{\bullet}\right)$.

Now we can prove the following general existence, uniqueness, and continuity theorem for abstract linear parabolic equations involving measures.

Theorem 8.1. Let assumptions $(\mathrm{H}),(6.1)$ and (8.1) be satisfied. Then:

(i) (Existence and uniqueness) Problem (8.2) has for each $\mu$ belonging to $\mathcal{M}\left([0, \mathrm{~T}), E_{1 / p^{\prime}, p}\right)$ a unique weak $L_{r}\left(E_{1}\right)$ solution $u$.

(ii) (Weak*-weak continuity) If $\mathcal{M}\left([0, \mathrm{~T}), E_{1 / p^{\prime}, p}\right)$ is given the weak star and $L_{r}\left(\mathrm{~J}, E_{1}\right)$ the weak topology, then the solution map

$$
\mathcal{M}\left([0, \mathrm{\top}), E_{1 / p^{\prime}, p}\right) \mapsto L_{r}\left((0, \mathrm{\top}), E_{1}\right), \quad \mu \mapsto u
$$

is continuous.

(iii) (Continuous dependence on all data) Suppose that

- $A_{j} \in \mathcal{M R}_{p}\left(\mathrm{~J},\left(E_{1}, E_{0}\right)\right)$ is such that $A_{j}^{\bullet} \in \mathcal{M R}_{p^{\prime}}\left(\mathrm{J},\left(E_{1}^{\bullet}, E_{0}^{\bullet}\right)\right)$;

- $B_{j} \in L_{1}\left(\mathrm{~J}, \mathcal{L}\left(E_{1}, E_{0}\right)\right) \cap \mathcal{L}\left(L_{r}\left(\mathrm{~J}, E_{1}\right), L_{1}\left(\mathrm{~J}, E_{1 / p^{\prime}, p}\right)\right)$;

- $\mu_{j} \in \mathcal{M}\left([0, \mathrm{~T}), E_{1 / p^{\prime}, p}\right)$;

- $\left(A_{j}^{\bullet}, B_{j}, \mu_{j}\right) \rightarrow\left(A^{\bullet}, B, \mu\right)$ in

$$
\begin{aligned}
\mathcal{L}\left(\mathbb{W}_{p^{\prime}, \mathrm{T}}^{1}\left(\mathrm{~J},\left(E_{1}^{\bullet}, E_{0}^{\bullet}\right)\right), L_{p^{\prime}}\left(\mathrm{J}, E_{0}^{\bullet}\right)\right) & \times \mathcal{L}\left(L_{r}\left(\mathrm{~J}, E_{1}\right), L_{1}\left(\mathrm{~J}, E_{1 / p^{\prime}, p}\right)\right) \\
& \times \mathcal{M}\left([0, \mathrm{~T}), E_{1 / p^{\prime}, p}\right) .
\end{aligned}
$$

Let $u_{j}$ be the weak $L_{r}\left(E_{1}\right)$ solution of $\dot{u}+\left(A_{j}+B_{j}\right) u=\mu_{j}$. Then $u_{j}$ converges in $L_{r}\left(\mathrm{~J}, E_{1}\right)$ towards $u$.

(iv) (Compactness) Suppose that $E_{1}$ is compactly embedded in $E_{0}$ and that $1 / p^{\prime}<\theta \leq 1$. Then the solution map is compact from $\mathcal{M}\left([0, \mathrm{~T}), E_{\theta, \infty}\right)$ into $L_{r}\left(\mathrm{~J}, E_{1}\right)$.

Proof. (i) Given $u \in L_{r}\left(\mathrm{~J}, E_{1 / p^{\prime}, p}\right)$, it follows from (7.2) that $-B u+\mu$ belongs to $\mathcal{M}\left([0, \mathrm{~T}), E_{1 / p^{\prime}, p}\right)$. Thus, setting

$$
C:=\left(K^{\bullet}\right)^{\prime} B \in \mathcal{L}\left(L_{r}\left(\mathrm{~J}, E_{1}\right)\right), \quad g:=\left(K^{\bullet}\right)^{\prime} \mu \in L_{r}\left(\mathrm{~J}, E_{1}\right),
$$

we deduce from (7.6) and (7.7) that (8.3) is equivalent to $u+C u=g$ in $L_{r}\left(\mathrm{~J}, E_{1}\right)$. Hence it suffices to show that $1+C$ is bijective. 
Note that (7.5) implies that $C \in L_{r}\left((0, T), E_{1}\right)$ and that there exists a constant $c_{0}$ such that

$$
\|C\|_{\mathcal{L}\left(L_{r}\left((0, T), E_{1}\right)\right)} \leq c_{0} T^{1 / r-1 / p}, \quad 0<T \leq \mathrm{T} .
$$

Fix $\tau \in(0, T]$ and a positive integer $k$ such that $c_{0} \tau^{1 / r-1 / p} \leq 1 / 2$ and $k \tau=\mathrm{T}$. Then we see from (8.4) that $(1+C)^{-1}$ exists in $\left.\mathcal{L}\left(L_{r}(0, \tau), E_{1}\right)\right)$ and has its norm bounded by 2 . Thus the assertion is true if $T$ is replaced by $(0, \tau)$. An obvious modification of this argument proves that (8.2) has a unique $L_{r}\left(E_{1}\right)$ solution if $[0, T)$ is replaced by $[i \tau,(i+1) \tau)$ for $1 \leq i \leq k-1$. Now the assertion follows from $\mu=\sum_{i=0}^{k-1} \mu \mid[i \tau,(i+1) \tau)$ and the linearity of the problem.

(ii) It suffices to prove the assertion if $[0, T)$ is replaced by a subinterval of length $\tau$. Thus we can assume that $1+C$ is an automorphism of $L_{r}\left(\mathrm{~J}, E_{1}\right)$. Hence, since $u=(1+C)^{-1}\left(K^{\bullet}\right)^{\prime} \mu$, it follows that the weak continuity of $u$ is equivalent to the weak continuity of $\left(K^{\bullet}\right)^{\prime} \mu$ in $L_{r}\left(\mathrm{~J}, E_{1}\right)$. Given $\varphi \in L_{r^{\prime}}\left(\mathrm{J}, E_{0}^{\bullet}\right)$, we know from (7.3) that $K^{\bullet} \varphi \in C_{0}\left([0, \mathrm{~T}), E_{1 / p, p^{\prime}}^{\bullet}\right)$. Now the assertion follows from

$$
\langle\varphi, u\rangle_{L_{r}\left(\mathrm{~J}, E_{1}\right)}=\left\langle\mu, K^{\bullet} \varphi\right\rangle_{C_{0}\left([0, \mathrm{~T}), E_{\mathbf{1}, p, p^{\prime}}\right)}
$$

and $\left(E_{1 / p, p^{\prime}}^{\bullet^{\prime}}\right)^{\prime}=E_{1 / p^{\prime}, p}($ see $(5.1))$.

(iii) The assumptions imply that

$$
-\partial+A_{j}^{\prime} \rightarrow-\partial+A \text { in } \mathcal{L}\left(\mathbb{W}_{p^{\prime}, \mathrm{T}}^{1}\left(\mathrm{~J},\left(E_{1}^{\bullet}, E_{0}^{\bullet}\right)\right), L_{p^{\prime}}\left(\mathrm{J}, E_{0}^{\bullet}\right)\right) .
$$

Thus the smoothness of the inversion map and $K_{j}^{\bullet}=\left(-\partial+A_{j}^{\prime}\right)^{-1}$ guarantee that $K_{j}^{\bullet} \rightarrow K^{\bullet}$ in $\mathcal{L}\left(L_{p^{\prime}}\left(\mathrm{J}, E_{0}^{\bullet}\right), \mathbb{W}_{p^{\prime}, \mathrm{T}}^{1}\left(\mathrm{~J},\left(E_{1}^{\bullet}, E_{0}^{\bullet}\right)\right)\right)$, hence, thanks to $(1.2)$ and $r^{\prime} \geq p^{\prime}$, in $\mathcal{L}\left(L_{r^{\prime}}\left(\mathrm{J}, E_{0}^{\bullet}\right), C_{0}\left([0, \mathrm{~T}), E_{1 / p, p^{\prime}}^{\bullet}\right)\right.$. Consequently, by invoking the isometry of the duality map, we see that $\left(K_{j}^{\bullet}\right)^{\prime} \rightarrow\left(K^{\bullet}\right)^{\prime}$ in $\mathcal{L}\left(\mathcal{M}\left([0, \mathrm{~T}), E_{1 / p^{\prime}, p}\right), L_{r}\left(\mathrm{~J}, E_{1}\right)\right)$. It follows that $C_{j}=\left(K^{\bullet}\right)^{\prime} B_{j} \rightarrow C$ in $\mathcal{L}\left(L_{r}\left(\mathrm{~J}, E_{1}\right)\right)$ (recall (7.2)). Since the set of automorphisms is open in $\mathcal{L}\left(L_{r}\left(\mathrm{~J}, E_{1}\right)\right)$ and inversion is smooth, we obtain $\left(1+C_{j}\right)^{-1} \rightarrow(1+C)^{-1}$ in $\mathcal{L}\left(L_{r}\left(\mathrm{~J}, E_{1}\right)\right)$. Now the assertion follows from $u_{j}=\left(1+C_{j}\right)^{-1}\left(K_{j}^{\bullet}\right)^{\prime} \mu_{j}$.

(iv) Schauder's theorem implies that $E_{1}^{\bullet}$ is compactly embedded in $E_{0}^{\bullet}$. Hence we deduce from (1.2) that $\mathbb{W}_{p^{\prime}, \mathrm{T}}^{1}\left(\mathrm{~J},\left(E_{1}^{\bullet}, E_{0}^{\bullet}\right)\right)$ embeds compactly into $C_{0}\left([0, \mathrm{~T}), E_{1-\theta, 1}^{\bullet}\right)$, thanks to $1-\theta<1 / p$. Thus $K^{\bullet}$ is a compact linear map from $L_{r^{\prime}}\left(\mathrm{J}, E_{0}^{\bullet}\right)$ into $C_{0}\left([0, \mathrm{~T}), E_{1-\theta, 1}^{\bullet}\right)$. Since

$$
\left(E_{1-\theta, 1}^{\bullet}\right)^{\prime}=\left(\left(E_{0}^{\bullet}, E_{1}^{\bullet}\right)_{1-\theta, 1}\right)^{\prime}=\left(E_{1}, E_{0}\right)_{1-\theta, \infty}=E_{\theta, \infty},
$$

Schauder's theorem guarantees that $\left(K^{\bullet}\right)^{\prime}$ maps $\mathcal{M}\left([0, T), E_{\theta, \infty}\right)$ compactly into $L_{r}\left(\mathrm{~J}, E_{1}\right)$.

\section{Positivity}

In this section we prove a general positivity result which will be used in the last section to derive a weak maximum principle for parabolic systems.

Suppose that $E$ is an ordered Banach space (OBS). Every Banach space $F$ satisfying $F \hookrightarrow E$, it is given the natural (or induced) order. Thus, if $E^{+}$is the positive cone of $E$ then $F^{+}:=i^{-1}\left(E^{+}\right)$, where $i: F \hookrightarrow E$ is 
the natural injection. The dual space $E^{\prime}$ is endowed with the dual order, that is, $e^{\prime} \in E^{\prime}$ is positive, in symbols: $e^{\prime} \geq 0$, iff $\left\langle e^{\prime}, e\right\rangle_{E} \geq 0$ for each $e \in E^{+}$. Clearly, $L_{1}(\mathrm{~J}, E)$ is given the natural order whose positive cone is $L_{1}\left(\mathrm{~J}, E^{+}\right)$. Product spaces of OBSs are ordered by the product of their positive cones.

An operator $B \in \mathcal{L}(E)$ is positive, that is, $B \geq 0$, iff $B e \geq 0$ for all $e \in E^{+}$. If $C$ is a closed linear operator in $E$ then $C$ is resolvent positive if some interval $(\alpha, \infty)$ belongs to the resolvent set of $-C$ and $(\lambda+C)^{-1}$ is positive for $\lambda>\alpha$. Finally, if $E_{0}$ is an OBS then $\mathcal{H}^{+}\left(E_{1}, E_{0}\right)$ denotes the set of all resolvent positive operators in $\mathcal{H}\left(E_{1}, E_{0}\right)$.

Theorem 9.1. Let $E_{0}$ be an $O B S$ and suppose that

$$
A \in \mathcal{M R}_{p}\left(\mathrm{~J},\left(E_{1}, E_{0}\right)\right) \cap C^{\rho}\left(\mathrm{J}, \mathcal{H}^{+}\left(E_{1}, E_{0}\right)\right)
$$

for some $\rho \in(0,1]$. Then $\left(\partial+A, \gamma_{0}\right)^{-1} \geq 0$.

Proof. Since $A \in C^{\rho}\left(\mathrm{J}, \mathcal{H}^{+}\left(E_{1}, E_{0}\right)\right)$ we know from [4, Theorem II.1.2.1] that (2.2) has for each $(f, x) \in C^{\rho}\left(\mathrm{J}, E_{0}\right) \times E_{1}$ a unique classical solution $u \in C^{1}\left(\mathrm{~J}, E_{1}\right)$. Furthermore, [4, Corollary II.4.4.2] guarantees the existence of a unique parabolic evolution operator $U$ (with regularity subspace $\left.E_{1}\right)$ such that

$$
u(t)=U(t, 0) x+\int_{0}^{t} U(t, \tau) f(\tau) d \tau, \quad t \in \mathrm{J} .
$$

Since $A(t) \in \mathcal{H}^{+}\left(E_{1}, E_{0}\right)$ we know from [4, Theorem II.6.4.2] that $U(t, s)$ is positive for $0 \leq s \leq t \leq \mathrm{T}$. Thus $u \geq 0$ if $(f, x) \geq 0$. Since $C^{1}\left(\mathrm{~J}, E_{1}\right)$ is a linear subspace of $\mathbb{W}_{p}^{1}\left(\mathrm{~J},\left(E_{1}, E_{0}\right)\right)$ it follows that $u=\left(\partial+A, \gamma_{0}\right)^{-1}(f, x)$. Hence

$$
\left(\partial+A, \gamma_{0}\right)^{-1}(f, x) \geq 0, \quad(f, x) \in C^{\rho}\left(\mathrm{J}, E_{0}^{+}\right) \times E_{1}^{+} .
$$

By mollification one shows that $C^{\rho}\left(\mathrm{J}, E_{0}^{+}\right)$is dense in $L_{p}\left(\mathrm{~J}, E_{0}^{+}\right)$. Put $B:=A(0)$. Then $(1+\varepsilon B)^{-1} x$ converges towards $x$ in $E_{1 / p^{\prime}, p}$ as $\varepsilon \rightarrow 0+$ (cf. Lemma II.6.1.1 and Theorem V.2.1.3 in [4]). From this, the fact that $(1+\varepsilon B)^{-1} x \in E_{1}$, and the resolvent positivity of $B$ we infer that $E_{1}^{+}$is dense in $E_{1 / p^{\prime}, p}^{+}$. Thus the assertion follows from (9.1) and the closedness of the positive cones.

\section{INTERPOLATION EXTRAPOLATION SETTINGS}

In this section we prove a version of Theorem 8.1 in a setting which is particularly well suited for applications to parabolic differential equations.

Suppose that

- $F_{0}$ is a reflexive Banach space;

- $C$ is a closed and densely defined linear operator in $F_{0}$ containing 0 in its resolvent set;

- $(\cdot, \cdot)_{\theta} \in\left\{[\cdot, \cdot]_{\theta},(\cdot, \cdot)_{\theta, r} ; 1<r<\infty\right\}$ for $0<\theta<1$.

Put $F_{1}:=D(C)$, the domain of $C$ endowed with its graph norm. Also put $F_{0}^{\sharp}:=F_{0}^{\prime}$ and $F_{1}^{\sharp}:=D\left(C^{\prime}\right)$. Denote by $(\cdot, \cdot)_{\theta}^{\sharp}$ the interpolation functor 
dual to $(\cdot, \cdot)_{\theta}$, defined by

$$
(\cdot, \cdot)_{\theta}^{\sharp}:= \begin{cases}{[\cdot, \cdot]_{\theta}} & \text { if }(\cdot, \cdot)_{\theta}=[\cdot, \cdot]_{\theta}, \\ (\cdot, \cdot)_{\theta, r^{\prime}} & \text { otherwise. }\end{cases}
$$

Set

$$
F_{\theta}:=\left(F_{0}, F_{1}\right)_{\theta}, \quad F_{\theta}^{\sharp}:=\left(F_{0}^{\sharp}, F_{1}^{\sharp}\right)_{\theta}, \quad 0<\theta<1,
$$

and

$$
F_{\theta}:=\left(F_{-\theta}^{\sharp}\right)^{\prime}, \quad F_{\theta}^{\sharp}:=\left(F_{-\theta}\right)^{\prime}, \quad-1 \leq \theta<0 .
$$

Then $\left[F_{\alpha} ;|\alpha| \leq 1\right]$ is the interpolation extrapolation (space) scale (of order 1$)$ generated by $(F, C)$ and $(\cdot, \cdot)_{\theta}, 0<\theta<1$, and $\left[F_{\alpha}^{\sharp} ;|\alpha| \leq 1\right]$ is its dual scale. It follows that $F_{\alpha}$ is reflexive with

$$
F_{\alpha} \doteq\left(F_{-\alpha}^{\sharp}\right), \quad|\alpha| \leq 1,
$$

where $\doteq$ means "equal except for equivalent norms". Moreover,

$$
F_{\alpha} \stackrel{d}{\hookrightarrow} F_{\beta}, \quad F_{\alpha}^{\sharp} \stackrel{d}{\hookrightarrow} F_{\beta}^{\sharp}, \quad-1 \leq \beta<\alpha \leq 1,
$$

and these embeddings are compact if $C$ has a compact inverse. (We refer to Chapter V in [4] for proofs and a detailed study of the interpolation extrapolation theory.)

Theorem 10.1. Let assumption (10.1) be satisfied. Suppose that

$$
0 \leq \alpha<\beta \leq 1, \quad 1 \leq r<p<1 /(1+\alpha-\beta) .
$$

Also suppose that

$$
A \in \mathcal{M R}_{p}\left(\mathrm{~J},\left(F_{\alpha}, F_{\alpha-1}\right)\right), \quad A^{\bullet} \in \mathcal{M R}_{p^{\prime}}\left(\mathrm{J},\left(F_{1-\alpha}^{\sharp}, F_{-\alpha}^{\sharp}\right)\right),
$$

and

$$
B \in L_{1}\left(\mathrm{~J}, \mathcal{L}\left(F_{\alpha}, F_{\alpha-1}\right)\right) \cap \mathcal{L}\left(L_{r}\left(\mathrm{~J}, F_{\alpha}\right), L_{1}\left(\mathrm{~J}, F_{\beta-1}\right)\right) .
$$

Then:

(i) (Existence and uniqueness) The problem

$$
\dot{u}+(A+B) u=\mu \quad \text { in }[0, T)
$$

has for each $\mu \in \mathcal{M}\left([0, T), F_{\beta-1}\right)$ a unique weak $L_{r}\left(F_{\alpha}\right)$ solution.

(ii) (Continuity) It possesses the continuity properties specified in (ii)-(iv) of Theorem 8.1 with $E_{1 / p^{\prime}, p}$ replaced by $F_{\beta-1}$.

(iii) (Compactness) If $F_{1}$ is compactly embedded in $F_{0}$, then the solution map is compact from $\mathcal{M}\left([0, \mathrm{~T}), F_{\beta-1}\right)$ into $L_{r}\left(\mathrm{~J}, F_{\alpha}\right)$.

Proof. Set $\left(E_{0}, E_{1}\right):=\left(F_{\alpha-1}, F_{\alpha}\right)$. Fix $\theta \in\left(1 / p^{\prime}, \beta-\alpha\right)$. Then the almost reiteration property of interpolation extrapolation scales from $[4$, Theorem V.1.5.3] and (1.1) imply

$$
F_{\beta-1} \hookrightarrow\left(F_{\alpha-1}, F_{\alpha}\right)_{\theta} \hookrightarrow E_{1 / p^{\prime}, p} .
$$

Hence

$$
\mathcal{M}\left([0, \mathrm{~T}), F_{\beta-1}\right) \hookrightarrow \mathcal{M}\left([0, \mathrm{~T}), E_{1 / p^{\prime}, p}\right),
$$

and the assertions follow from Theorem 8.1 and the fact that $C$ has a compact resolvent because of the compact embedding of $F_{1}$ in $F_{0}$. 
Remark 10.2. Let $F_{0}$ be a reflexive Banach space and $A \in \mathcal{H}\left(F_{0}\right)$. Fix a positive $\omega$ in the resolvent set of $-A_{0}$ and put $C:=\omega+A_{0}$. Then $C$ satisfies the assumptions specified in (10.1). For $0 \leq \xi<1$ let $A_{\xi-1}$ be the closure of $A_{0}$ in $F_{\xi-1}$, and denote by $A_{-\xi}^{\sharp}$ the closure of $A_{0}^{\sharp}$ in $F_{-\xi}^{\sharp}$. Then $A_{1-\xi}$ and $A_{-\xi}^{\sharp}$ are well-defined and

$$
A_{\xi-1} \in \mathcal{H}\left(F_{\xi}, F_{\xi-1}\right), \quad A_{-\xi}^{\sharp} \in \mathcal{H}\left(F_{1-\xi}^{\sharp}, F_{-\xi}^{\sharp}\right), \quad A_{-\xi}^{\sharp}=\left(A_{\xi-1}\right)^{\prime}
$$

(cf. [4, Chapter V]). Thus, setting $A:=A_{\alpha-1}$, assumption (10.2) reduces to

$$
A_{\alpha-1} \in \mathcal{M R}_{p}\left(F_{\alpha}, F_{\alpha-1}\right), \quad A_{-\alpha}^{\sharp} \in \mathcal{M R}_{p^{\prime}}\left(F_{1-\alpha}^{\sharp}, F_{-\alpha}^{\sharp}\right) .
$$

Given these additional hypotheses, Theorems 4.2 and 10.1 generalize the abstract results of [5] and simplify their proofs.

\section{NONLinear PROBLEMS}

In [10] the results of [5] were used to derive existence, uniqueness, and continuity theorems for solutions of semilinear problems of the form

$$
\dot{u}+A u=\mu(u) \quad \text { in }[0, T)
$$

in the setting of Remark 10.2. Here $\mu(u) \in \mathcal{M}\left([0, T), F_{\beta-1}\right)$ depends Lipschitz continuously on $u$ in suitable topologies, and possesses the Volterra property. The proofs are solely based on the existence theory for linear problems and on estimates of the type contained in Theorem 4.2. Thus, given assumption (10.2), we obtain the complete analogues to Theorems 1.1 and 1.2 as well as Theorems 2.1-2.3 of [10]. In fact, since we can now consider measures in $\mathcal{M}\left([0, T), E_{\beta-1}\right)$ (instead of those belonging to $\left.\mathcal{M}\left([0, T], E_{\beta-1}\right)\right)$ for $0<T \leq \mathrm{T}$, the proof of [10, Theorem 1.1] simplifies since the "gluing of the solutions" (see [10, Section 12]) is now trivial (cf. the proof of Theorem 8.1). We leave the details to the interested reader.

\section{Part 2. Parabolic systems}

In this part we apply the abstract results of the preceding part to parabolic boundary value problems. We restrict ourselves to second order problems, but consider strongly coupled systems. We leave it to the the interested reader to derive similar results for higher order equations.

Throughout this part we fix $N \in \mathbb{N} \backslash\{0\}$ and suppose that $1<q<\infty$. We assume that $\Omega$ is a smooth subdomain of $\mathbb{R}^{n}$ having a compact boundary $\Gamma$. Of course, in the following all explicit or implicit references to $\Gamma$ have to be neglected if $\Omega=\mathbb{R}^{n}$.

Let $M$ be a submanifold of $\mathbb{R}^{n}$ and $F$ a Banach space. Suppose that $X(M, F)$ is a space of distributions on $M$ with values in $F$. Then we write simply $X(M)$ for $X\left(M, \mathbb{R}^{N}\right)$ and $X:=X(M)$. Moreover, $X\left(M, \mathbb{R}^{N \times N}\right)$ is denoted by $\boldsymbol{X}(M)$, and $\boldsymbol{X}:=\boldsymbol{X}(\Omega)$. In all other cases we mention explicitly $M$ or $F$. Thus, for example, $\mathcal{D}$ is the space of $\mathbb{R}^{N}$-valued distributions in $\Omega$, and $W_{p}^{s}(\Gamma)$ the standard Sobolev-Slobodeckii space of $\mathbb{R}^{N}$-valued distributions on $\Gamma$. Furthermore, $\boldsymbol{B U} \boldsymbol{C}$ is the space of all $(N \times N)$-matrix valued functions on $\Omega$ which are bounded and uniformly 
continuous. Of course, we identify $a \in \boldsymbol{B} \boldsymbol{U} \boldsymbol{C}$ with its unique extension in $\boldsymbol{B U \boldsymbol { C }}(\bar{\Omega})$. Clearly, $a \in \boldsymbol{B} \boldsymbol{U} \boldsymbol{C}^{1}$ iff $a$ and $\partial_{j} a$ belong to $\boldsymbol{B U \boldsymbol { C }}$ for $1 \leq j \leq n$.

\section{Normally Elliptic boundary Value Problems}

Suppose that

$$
a_{j k} \in \boldsymbol{B U C}, \quad 1 \leq j, k \leq n
$$

Put

$$
\mathcal{A}:=-\partial_{j}\left(a_{j k} \partial_{k} \cdot\right)
$$

and denote the principal symbol of $\mathcal{A}$ by $a_{\pi}$, that is,

$$
a_{\pi}(x, \xi):=a_{j k}(x) \xi^{j} \xi^{k} \in \mathbb{R}^{N \times N}, \quad(x, \xi) \in \Omega \times \mathbb{R}^{n} .
$$

Then $\mathcal{A}$ is said to be normally elliptic if there exists $\varepsilon \in(0,1]$ such that

$$
\sigma\left(a_{\pi}(x, \xi)\right) \subset[\operatorname{Re} z \geq \varepsilon,|z| \leq 1 / \varepsilon], \quad(x, \xi) \in \Omega \times S^{n-1},
$$

where $\sigma(\cdot)$ denotes the spectrum, $S^{n-1}$ is the unit sphere in $\mathbb{R}^{n}$, and $[\ldots]$ is the subset of $\mathbb{C}$ defined by the conditions listed therein.

We fix a diagonal matrix valued function $\chi=\operatorname{diag}\left[\chi^{1}, \ldots, \chi^{N}\right] \in \boldsymbol{C}(\Gamma)$ satisfying

$$
\chi^{r}(y) \in\{0,1\}, \quad y \in \Gamma, \quad 1 \leq r \leq N,
$$

a boundary characterization map for $\Omega$. Note that, given $r \in\{1, \ldots, N\}$, the sets $\Gamma_{i}^{r}:=\left(\chi^{r}\right)^{-1}(i), i \in\{0,1\}$, are open in $\Gamma$, disjoint, and have $\Gamma$ as their union.

For $u \in C^{1}(\Gamma)$ we also set

$$
\partial_{\nu} u:=\nu^{j} \gamma\left(a_{j k} \partial_{k} u\right) \in C(\Gamma),
$$

where $\nu=\left(\nu^{1}, \ldots, \nu^{n}\right)$ is the outer unit normal and $\gamma$ the trace operator for $\Gamma$. We define a boundary operator by

$$
\mathcal{B}:=\chi \partial_{\nu}+(1-\chi) \gamma
$$

Observe that the $r$-th component of $\mathcal{B} u$ is the Dirichlet boundary operator on $\Gamma_{0}^{r}$ for the $r$-th component of $u$, whereas it is a Neumann boundary operator on $\Gamma_{1}^{r}$, involving, in general, all components of $u$.

We denote the symbol of $\mathcal{B}$ by $b_{\pi}$, that is,

$$
b_{\pi}(y, \xi):=\chi(y) \nu^{j}(y) a_{j k}(y) \xi^{k}+(1-\chi(y)), \quad(y, \xi) \in \Gamma \times \mathbb{R}^{n} .
$$

Then $\mathcal{B}$ satisfies the parabolic (or parameter dependent) Lopatinskii Shapiro conditions with respect to $\mathcal{A}$ if, for each $((y, \xi), \lambda) \in T \Gamma \times[\operatorname{Re} z \geq 0]$ with $(\xi, \lambda) \neq(0,0)$, zero is the only exponentially decaying solution of the boundary value problem on the half line:

$$
\left[\lambda+a_{\pi}\left(y, \xi+\nu(y) i \partial_{t}\right)\right] v=0, \quad t>0, \quad b_{\pi}\left(y, \xi+\nu(y) i \partial_{t}\right) v(0)=0,
$$

$T \Gamma$ being the tangent bundle of $\Gamma$. The second order linear boundary value problem $(\mathcal{A}, \mathcal{B})$ on $\Omega$ is called normally elliptic if $\mathcal{A}$ is normally elliptic and $\mathcal{B}$ satisfies the parabolic Lopatinskii Shapiro conditions with respect to $\mathcal{A}$. We refer to [3, Examples 4.3] for a variety of simple sufficient conditions on $(\mathcal{A}, \mathcal{B})$ guaranteeing that it is normally elliptic.

The formally dual boundary value problem $\left(\mathcal{A}^{\sharp}, \mathcal{B}^{\sharp}\right)$ of $(\mathcal{A}, \mathcal{B})$ is obtained by replacing $a_{j k}$ by the transposed matrix $a_{k j}^{\top}$ for $1 \leq j, k \leq n$. It is normally elliptic iff $(\mathcal{A}, \mathcal{B})$ has this property. 
We denote by

$$
\mathcal{E} \ell \ell^{1}(\Omega):=\mathcal{E} \ell \ell^{1}(\Omega, \chi, N)
$$

the set of all $a_{j k} \in \boldsymbol{B} \boldsymbol{U} \boldsymbol{C}^{1}, \quad 1 \leq j, k \leq n$, such that $(\mathcal{A}, \mathcal{B})$, defined by (12.1) and (12.3), is a normally elliptic boundary value problem on $\Omega$. In abuse of notation we write $(\mathcal{A}, \mathcal{B}) \in \mathcal{E} \ell \ell^{1}(\Omega)$ in this case. It can be shown that $\mathcal{E} \ell \ell^{1}(\Omega)$ is open in $\left(\boldsymbol{B} \boldsymbol{U} \boldsymbol{C}^{1}\right)^{n \times n}$ (see [2, Theorem 2]).

Suppose that $(\mathcal{A}, \mathcal{B}) \in \mathcal{E} \ell \ell^{1}(\Omega)$. We set

$$
W_{q, \mathcal{B}}^{s}:=\left\{\begin{array}{cc}
\left\{u \in W_{q}^{s} ; \mathcal{B} u=0\right\}, & 1+1 / q<s \leq 2, \\
\left\{u \in W_{q}^{s} ;(1-\chi) \gamma u=0\right\}, & 1 / q<s<1+1 / q, \\
W_{q}^{s}, & 0 \leq s<1 / q, \\
\left(W_{q^{\prime}, \mathcal{B} \sharp}^{-s}\right)^{\prime}, & -2 \leq s<0, \quad s \notin \mathbb{Z}+1 / q,
\end{array}\right.
$$

where the dual space is determined by the duality pairing naturally induced by

$$
\langle u, v\rangle:=\int_{\Omega} u \cdot v d x, \quad u, v \in \mathcal{D} .
$$

For each $s \in[0,2] \backslash(\mathbb{N}+1 / q)$ we define a linear operator

$$
A_{s-2} \in \mathcal{L}\left(W_{q, \mathcal{B}}^{s}, W_{q, \mathcal{B}}^{s-2}\right),
$$

the $W_{q, \mathcal{B}}^{s-2}$ realization of $(\mathcal{A}, \mathcal{B})$ as follows:

- if $1+1 / q<s \leq 2$ then $A_{s-2}:=\mathcal{A} \mid W_{q, \mathcal{B}}^{s}$;

- if $1 / q<s<1+1 / q$ then

$$
\left\langle v, A_{s-2} u\right\rangle_{W_{q, \mathcal{B}}^{s-2}}:=\left\langle\partial_{j} v, a_{j k} \partial_{k} u\right\rangle, \quad(v, u) \in W_{q^{\prime}, \mathcal{B}^{\sharp}}^{2-s} \times W_{q, \mathcal{B}}^{s},
$$

- $\quad$ if $0 \leq s<1 / q$ then

$$
\left\langle v, A_{s-2} u\right\rangle_{W_{q, \mathcal{B}}^{s-2}}:=\left\langle\mathcal{A}^{\sharp} v, u\right\rangle, \quad(v, u) \in W_{q^{\prime}, \mathcal{B}}^{2-s} \times W_{q, \mathcal{B}}^{s} .
$$

Similarly,

$$
A_{s-2}^{\sharp} \in \mathcal{L}\left(W_{q^{\prime}, \mathcal{B}}^{s}, W_{q^{\prime}, \mathcal{B}^{\sharp}}^{s-2}\right),
$$

the $W_{q^{\prime}, \mathcal{B}^{\sharp}}^{s-2}$ realization of $\left(\mathcal{A}^{\sharp}, \mathcal{B}^{\sharp}\right)$, is defined by replacing in the preceding definitions $(\mathcal{A}, \mathcal{B})$ by $\left(\mathcal{A}^{\sharp}, \mathcal{B}^{\sharp}\right)$ and $q$ by $q^{\prime}$. (Note that $\left(\left(\mathcal{A}^{\sharp}\right)^{\sharp},\left(\mathcal{B}^{\sharp}\right)^{\sharp}\right)$ equals $(\mathcal{A}, \mathcal{B})$.)

Theorem 12.1. Suppose that $(\mathcal{A}, \mathcal{B}) \in \mathcal{E} \ell \ell^{1}(\Omega)$. Then

(i) $A_{s-2} \in \mathcal{H}\left(W_{q, \mathcal{B}}^{s}, W_{q, \mathcal{B}}^{s-2}\right)$ for $s \in[0,2] \backslash(\mathbb{N}+1 / q)$.

(ii) $W_{q, \mathcal{B}}^{s}$ is reflexive and $\left(W_{q, \mathcal{B}}^{s}\right)^{\prime} \doteq W_{q^{\prime}, \mathcal{B}}^{-s}$ for $s \in[-2,2] \backslash(\mathbb{Z}+1 / q)$.

(iii) $\left(A_{s-2}\right)^{\prime}=A_{-s}^{\sharp}$ for $s \in[0,2] \backslash(\mathbb{N}+1 / q)$.

(iv) Fix $\omega>0$ in the resolvent set of $-A$ and let $\left[F_{\alpha} ;|\alpha| \leq 1\right]$ be the interpolation extrapolation scale generated by $\left(L_{q}, \omega+\bar{A}_{0}\right)$ and $(\cdot, \cdot)_{\theta}, \quad 0<\theta<1$, where

$$
(\cdot, \cdot)_{\theta}:= \begin{cases}{[\cdot, \cdot]_{1 / 2}} & \text { if } \theta=1 / 2 \\ (\cdot, \cdot)_{\theta, q} & \text { otherwise. }\end{cases}
$$

Then

$$
F_{\alpha} \doteq W_{q, \mathcal{B}}^{2 \alpha}, \quad 2 \alpha \in[-2,2] \backslash(\mathbb{Z}+1 / q),
$$


and

$$
F_{\alpha}^{\sharp} \doteq W_{q^{\prime}, \mathcal{B}^{\sharp}}^{2 \alpha}, \quad 2 \alpha \in[-2,2] \backslash\left(\mathbb{Z}+1 / q^{\prime}\right) .
$$

Proof. The fact that assertion (i) is true for $s=2$ is more or less wellknown. For example, see [1] (for elliptic systems of arbitrary even order in domains whose boundary is not necessarily compact), [2] (for bounded domains), [8] (for elliptic operators of arbitrary order on $\mathbb{R}^{N}$ ), and, more recently, [13] (where the case of elliptic boundary value problems of arbitrary even order with operator valued coefficients is treated, assuming that the top-order coefficients have a limit if $|x| \rightarrow \infty$ in the case of an unbounded $\Omega$ ). The remaining assertions follow from the theory of interpolation extrapolation spaces (see [3]).

It should be remarked that the assumption that $\mathcal{A}$ be normally elliptic is necessary for $A$ to belong to $\mathcal{H}\left(W_{q, \mathcal{B}}^{2}, L_{q}\right)$ (see [2]).

The following maximal regularity theorem is the basis for our further considerations.

Theorem 12.2. If $(\mathcal{A}, \mathcal{B}) \in \mathcal{E} \ell \ell^{1}(\Omega)$ then

$$
A_{s-2} \in \mathcal{M R}_{p}\left(W_{q, \mathcal{B}}^{s}, W_{q, \mathcal{B}}^{s-2}\right), \quad s \in[0,2] \backslash(\mathbb{N}+1 / q) .
$$

Proof. Since $L_{q}$ is a UMD space, $W_{q, \mathcal{B}}^{-2}$ is also such a space, being isomorphic to $L_{q}$. Thus $W_{q, \mathcal{B}}^{s-2}$ is a UMD space as well. (See Section III.4.4 and, in particular, Theorem III.4.5.2 in [4].) Thus it remains to show that $A_{s-2} \in \mathcal{B I P}\left(W_{q, \mathcal{B}}^{s}, W_{q, \mathcal{B}}^{s-2}\right)$. Because of Theorem 12.1 and [4, Proposition V.1.5.5], it suffices to show this for $s=2$. In that case it follows by obvious modifications of the proof of [12, Theorem 8], provided $\Omega$ is bounded. If $\Omega=\mathbb{R}^{n}$, it is a special case of [8, Corollary 9.7]. If $\Gamma \neq \emptyset$ and $\Omega$ is unbounded one has to adapt the localization argument of $[12$, Section 8] by a modification of the one used in [8], to avoid the hypothesis that $a_{j k}$ has a limit as $|x| \rightarrow \infty$. Details will be given elsewhere.

\section{LOWER ORDER TERMS}

Put $Q:=(-1,1)^{n}$. Suppose that $1 \leq p \leq \infty$. Then $a \in \boldsymbol{L}_{1, \text { loc }}\left(\mathbb{R}^{n}\right)$ is said to be in $L_{p}$ locally uniformly if

$$
\|a\|_{p, \text { unif }}:=\sup _{x \in \mathbb{Z}^{n}}\|a\|_{\boldsymbol{L}_{p}(x+Q)} .
$$

For $a \in \boldsymbol{L}_{1, \text { loc }}$ we write $a^{0}$ for its trivial extension over $\mathbb{R}^{N}$. Then we set

$$
\boldsymbol{L}_{p, \text { unif }}:=\left\{a \in \boldsymbol{L}_{1, \text { loc }} ;\left\|a^{0}\right\|_{p, \text { unif }}<\infty\right\},
$$

equipped with the norm

$$
a \mapsto\|a\|_{p, \text { unif }}:=\left\|a^{0}\right\|_{p, \text { unif }} .
$$

Note that $\boldsymbol{L}_{p, \text { unif }}$ is a Banach space, and $\boldsymbol{L}_{p \text {,unif }}=L_{p}$ if $\Omega$ is bounded. Furthermore,

$$
\boldsymbol{L}_{p}+\boldsymbol{L}_{\infty} \hookrightarrow \boldsymbol{L}_{p, \text { unif }} \hookrightarrow \boldsymbol{L}_{q \text {, unif }}
$$

for $1 \leq q<p \leq \infty$. It is not difficult to see that

$$
\boldsymbol{B} \boldsymbol{U} \boldsymbol{C}_{\Gamma}^{1} \stackrel{d}{\hookrightarrow} \boldsymbol{L}_{p, \text { unif }}, \quad 1 \leq p<\infty,
$$


where the space on the left-hand side consists of all $a \in \boldsymbol{B} \boldsymbol{U} \boldsymbol{C}^{1}$ vanishing on $\Gamma$.

Proposition 13.1. Suppose that $p, q, r \in[1, \infty)$ satisfy $1 / p+1 / q=1 / r$. Then the map

$$
\boldsymbol{L}_{p, \text { unif }} \times L_{q} \rightarrow L_{r}, \quad(a, u) \mapsto a u
$$

is well-defined, bilinear, and continuous.

Proof. If $\Omega$ is bounded, so that $\boldsymbol{L}_{p, \text { unif }}=\boldsymbol{L}_{p}$, then this follows from Hölder's inequality. Otherwise fix an enumeration $\left(U_{j}\right)$ of the open covering

$$
\left\{(x+Q) \cap \Omega ; x \in \mathbb{Z}^{n},(x+Q) \cap \Omega \neq \emptyset\right\}
$$

of $\Omega$. Choose smooth functions $\pi_{j}$ with support in $U_{j}$ such that $\sup _{j}\left\|\pi_{j}\right\|_{\infty}$ is finite and $\left(\pi_{j}^{2}\right)$ is a partition of unity on $\Omega$. Then $u \mapsto\left(\sum_{j}\left\|\pi_{j} u\right\|_{\alpha}^{\alpha}\right)^{1 / \alpha}$ is an equivalent norm for $L_{\alpha}$ whenever $1 \leq \alpha<\infty$ (cf. Section 9 in [8]). Since

$$
\left\|\pi_{j} a u\right\|_{r} \leq\|a\|_{p, \text { unif }}\left\|\pi_{j} v\right\|_{q}, \quad j \in \mathbb{N}
$$

the assertion is obvious.

In the remainder of this paper $q$ and $s$ are real numbers, and $\xi, \eta$, and $\zeta$ belong to $[1, \infty]$.

First we suppose that

$$
\left.\begin{array}{l}
\text { - } 0 \leq s<1-\frac{n}{q^{\prime}} ; \\
\text { - } \frac{1}{\xi} \leq \frac{s}{n}+\frac{1}{q^{\prime}} .
\end{array}\right\}
$$

Then we put

$$
\mathbb{L}:=\left(\boldsymbol{L}_{\xi, \text { unif }}\right)^{n+1}
$$

and, given $\boldsymbol{\alpha}:=\left(a_{1}, \ldots, a_{n}, a_{0}\right) \in \mathbb{L}$, set

$$
b(\boldsymbol{\alpha}, v, u):=\sum_{j=1}^{n}\left\langle\partial_{j} v, a_{j} u\right\rangle+\left\langle v, a_{0} u\right\rangle .
$$

We also consider the following hypothesis:

- $1 \leq s<2-\frac{n}{q^{\prime}}$

- $\frac{1}{\xi} \leq \frac{s}{n}+\frac{1}{q^{\prime}}$

- $\frac{1}{\eta} \leq \frac{s-1}{n}+\frac{1}{q^{\prime}}$;

In this case we set

- $\frac{1}{\zeta} \leq \frac{n}{n-1}\left(\frac{s-1}{n}+\frac{1}{q^{\prime}}\right)$.

$$
\mathbb{L}:=\left(\boldsymbol{L}_{\eta, \text { unif }}\right)^{2 n} \times \boldsymbol{L}_{\xi, \text { unif }} \times \boldsymbol{L}_{\zeta}(\Gamma)
$$

and, given $\boldsymbol{\alpha}:=\left(a_{1}, \ldots, a_{n}, c_{1}, \ldots, c_{n}, a_{0}, b\right) \in \mathbb{L}$,

$$
b(\boldsymbol{\alpha}, v, u):=\sum_{j=1}^{n}\left\{\left\langle\partial_{j} v, a_{j} u\right\rangle+\left\langle v, c_{j} \partial_{j} u\right\rangle\right\}+\left\langle v, a_{0} u\right\rangle+\langle\gamma v, \chi b \gamma u\rangle_{\Gamma}
$$


where

$$
\langle\varphi, \psi\rangle_{\Gamma}:=\int_{\Gamma} \varphi \cdot \psi d \sigma, \quad \varphi, \psi \in L_{1}(\Gamma),
$$

provided $\varphi \cdot \psi$ is integrable with respect to the volume measure $d \sigma$ of $\Gamma$.

Lemma 13.2. Suppose that either hypothesis

$$
\text { (13.2) is satisfied and } s<\sigma<1-n / q^{\prime} \text {, }
$$

or

$$
\text { (13.4) is satisfied and } s<\sigma<2-n / q^{\prime} \text {. }
$$

Then $b$ is a well-defined continuous trilinear form on $\mathbb{L} \times W_{q^{\prime}}^{2-\sigma} \times W_{q}^{s}$.

Proof. (i) Let (13.2) be satisfied. Since $W_{q}^{s} \hookrightarrow L_{\xi^{\prime}}$, it follows from Proposition 13.1 that

$$
\boldsymbol{L}_{\xi, \text { unif }} \times W_{q}^{s} \rightarrow L_{1}, \quad(a, u) \mapsto a u
$$

is bilinear and continuous. Thus, since $W_{q^{\prime}}^{2-\sigma} \hookrightarrow L_{\infty}$,

$$
(a, v, u) \mapsto\langle v, a u\rangle
$$

is a continuous trilinear form on $\boldsymbol{L}_{\xi \text {, unif }} \times W_{q^{\prime}}^{2-\sigma} \times W_{q}^{s}$. An obvious modification of this argument shows that $(a, v, u) \mapsto\left\langle\partial_{j} v, a u\right\rangle$ is a continuous trilinear form on this space. This proves the assertion if (13.2) is true.

(ii) If (13.4) is satisfied, then a similar reasoning invoking, in addition, the trace theorem, gives the assertion.

Let the hypothesis of Lemma 13.2 be satisfied. For $\boldsymbol{\alpha} \in \mathbb{L}$ define

$$
B(\boldsymbol{\alpha}) \in \mathcal{L}\left(W_{q, \mathcal{B}}^{s}, W_{q, \mathcal{B}}^{\sigma-2}\right) \quad \text { and } \quad C(\boldsymbol{\alpha}) \in \mathcal{L}\left(W_{q^{\prime}, \mathcal{B}^{\sharp}}^{2-\sigma}, W_{q^{\prime}, \mathcal{B}^{\sharp}}^{-s}\right)
$$

by

$$
\langle v, B(\boldsymbol{\alpha}) u\rangle_{W_{q, \mathcal{B}}^{\sigma-2}}:=b(\boldsymbol{\alpha}, v, u)=:\langle C(\boldsymbol{\alpha}) v, u\rangle_{W_{q, \mathcal{B}}^{s}}
$$

for $(v, u) \in W_{q^{\prime}, \mathcal{B}^{\sharp}}^{2-\sigma} \times W_{q, \mathcal{B}}^{s}$. Then it follows from that lemma and Theorem 12.1(ii) that $B(\boldsymbol{\alpha})$ and $C(\boldsymbol{\alpha})$ are well-defined and that

$$
(\boldsymbol{\alpha} \mapsto B(\boldsymbol{\alpha})) \in \mathcal{L}\left(\mathbb{L}, \mathcal{L}\left(W_{q, \mathcal{B}}^{s}, W_{q, \mathcal{B}}^{\sigma-2}\right)\right)
$$

and

$$
(\boldsymbol{\alpha} \mapsto C(\boldsymbol{\alpha})) \in \mathcal{L}\left(\mathbb{L}, \mathcal{L}\left(W_{q^{\prime}, \mathcal{B}^{\sharp}}^{2-\sigma}, W_{q^{\prime}, \mathcal{B}^{\sharp}}^{-s}\right)\right) .
$$

\section{The General CASE}

Now we formulate our assumptions for the nonautonomous case. We suppose that

- $0<T<\infty$;

- $a_{j k} \in C\left([0, T], \boldsymbol{B} \boldsymbol{U} \boldsymbol{C}^{1}\right), 1 \leq j, k \leq n$;

- $\chi$ is a boundary characterization map for $\Omega$.

Then we put

$$
\mathcal{A}_{\pi}:=-\partial_{j}\left(a_{j k} \partial_{k} \cdot\right), \quad \mathcal{B}_{\pi}:=\chi \partial_{\nu}+(1-\chi) \gamma
$$

and suppose that

$$
\left(\mathcal{A}_{\pi}(t), \mathcal{B}_{\pi}(t)\right) \in \mathcal{E} \ell \ell^{1}(\Omega), \quad t \in[0, T]
$$


We also set

$$
W_{q, \mathcal{B}}^{\alpha}:=W_{q, \mathcal{B}_{\pi}(0)}^{\alpha}, \quad W_{q^{\prime}, \mathcal{B}^{\sharp}}^{\beta}:=W_{q^{\prime}, \mathcal{B}_{\pi}^{\sharp}(0)}^{\beta} .
$$

for $\alpha, \beta \in[-2,2]$ with $\alpha \notin \mathbb{Z}+1 / q$ and $\beta \notin \mathbb{Z}+1 / q^{\prime}$.

$$
\text { THE CASE } 0 \leq s<1-n / q^{\prime}
$$

In addition to (14.1) and (14.2) we suppose that

- assumption (13.2) is satisfied;

- $W_{q, \mathcal{B}_{\pi}(t)}^{2} \doteq W_{q, \mathcal{B}}^{2}, W_{q^{\prime}, \mathcal{B}_{\pi}^{\sharp}(t)}^{2} \doteq W_{q^{\prime}, \mathcal{B}^{\sharp}}^{2}$ for $t \in[0, T]$;

- $\left.\boldsymbol{\alpha}:=\left(a_{1}, \ldots, a_{n}, a_{0}\right) \in L_{1}(0, T), \mathbb{L}\right)$,

where $\mathbb{L}$ is defined by (13.3).

Remarks 14.1. (a) The second condition of (14.3) is satisfied, that is, the spaces $W_{q, \mathcal{B}_{\pi}(t)}^{2}$ and $W_{q^{\prime}, \mathcal{B}_{\pi}^{\sharp}(t)}^{2}$ are independent of $t \in[0, T]$, if one of the following conditions is satisfied:

(i) $\chi=0$, that is, $\mathcal{B}_{\pi}$ is the pure Dirichlet boundary operator: $\mathcal{B}_{\pi}=\gamma$.

(ii) $a_{j k}$ is independent of $t$ for $1 \leq j, k \leq n$.

(iii) $a_{j k}=\mathrm{A} \alpha_{j k}$ for $1 \leq j, k \leq n$, where

$\mathrm{A} \in C\left([0, T], \boldsymbol{B U} \boldsymbol{C}^{1}\right), \quad\left[\alpha_{j k}\right] \in B U C^{1}\left(\Omega, \mathbb{R}^{n \times n}\right)$, $\left[\alpha_{j k}\right]$ is symmetric and uniformly positive definit, $\chi \in C\left(\Gamma,\left\{0,1_{N}\right\}\right)$.

Proof. (i) and (ii) are clear. (iii) In this case, given any component $\widetilde{\Gamma}$ of $\Gamma$, either $\chi \mid \widetilde{\Gamma}=0$, that is, $\mathcal{B}_{\pi}=\mathcal{B}_{\pi}^{\sharp}=\gamma$ on $\widetilde{\Gamma}$, or $\chi \mid \widetilde{\Gamma}=1_{N}$ and $\mathcal{B}_{\pi} u=0$ $\left[\right.$ resp. $\left.\mathcal{B}_{\pi}^{\sharp} v=0\right]$ is equivalent to $\partial_{\nu_{\alpha}} u=0\left[\operatorname{resp} . \partial_{\nu_{\alpha}} v=0\right]$, where $\nu_{\alpha}$ is the conormal with respect to $\left[\alpha_{j k}\right]$.

(b) Suppose that $W_{q, \mathcal{B}_{\pi}(t)}^{2} \doteq W_{q, \mathcal{B}}^{2}$ and $W_{q^{\prime}, \mathcal{B}_{\pi}^{\sharp}(t)}^{2} \doteq W_{q^{\prime}, \mathcal{B}^{\sharp}}^{2}$. Then

$$
W_{q, \mathcal{B}_{\pi}(t)}^{\alpha} \doteq W_{q, \mathcal{B}}^{\alpha} \quad \text { and } \quad W_{q^{\prime}, \mathcal{B}_{\pi}^{\sharp}(t)}^{\beta} \doteq W_{q^{\prime}, \mathcal{B}^{\sharp}}^{\beta}
$$

for all $\alpha \in[-2,2] \backslash(\mathbb{Z}+1 / q)$ and $\beta \in[-2,2] \backslash\left(\mathbb{Z}+1 / q^{\prime}\right)$.

Proof. This follows from interpolation characterizations of $W_{q, \mathcal{B}_{\pi}(t)}^{\alpha}$ and $W_{q^{\prime}, \mathcal{B}_{\pi}(t)}^{\beta}$ if $\alpha, \beta \geq 0$ (cf. [3]), and from Theorem 12.1(ii) if $\alpha, \beta<0$. by

We define the nonautonomous boundary value problem $(\mathcal{A}, \mathcal{B})$ formally

$$
\mathcal{A} u:=-\partial_{j}\left(a_{j k} \partial_{k} u+a_{j} u\right)+a_{0} u
$$

and

$$
\mathcal{B} u:=\chi \partial_{\nu} u+(1-\chi) \gamma u
$$

Fix $\sigma$ satisfying

$$
s<\sigma<1-n / q^{\prime} .
$$

Let $A(t):=A_{s-2}(t)$ be the $W_{q, \mathcal{B}}^{s-2}$ realization of $\left(\mathcal{A}_{\pi}(t), \mathcal{B}_{\pi}(t)\right)$, and define

$$
B(t):=B(\boldsymbol{\alpha}(t)) \in \mathcal{L}\left(W_{q, \mathcal{B}}^{s}, W_{q, \mathcal{B}}^{\sigma-2}\right)
$$


by (13.6). Then $(\mathcal{A}(t), \mathcal{B}(t))$ is said to be the $W_{q, \mathcal{B}}^{s-2}$ realization of $(\mathcal{A}(t), \mathcal{B}(t))$. Note that

$$
\langle v,(A(t)+B(t)) u\rangle_{W_{q, \mathcal{B}}^{s-2}}=\left\langle-\partial_{k}\left(a_{j k}^{\top}(t) \partial_{j} v\right)+a_{j}^{\top}(t) \partial_{j} v+a_{0}^{\top}(t) v, u\right\rangle
$$

for $(v, u) \in W_{q^{\prime}, \mathcal{B}^{\sharp}}^{2-s} \times W_{q, \mathcal{B}}^{s}$.

\section{The CASe $1 \leq s<2-n / q^{\prime}$}

Now we consider, in addition to (14.1) and (14.2), the hypothesis

- assumption (13.4) is satisfied;

- $\left.\alpha:=\left(a_{1}, \ldots, a_{n}, c_{1}, \ldots, c_{n}, a_{0}, b\right) \in L_{1}((0, T), \mathbb{L}), \quad\right\}$

where $\mathbb{L}$ is given by (13.5).

In this case we define $(\mathcal{A}, \mathcal{B})$ formally by

$$
\mathcal{A} u:=-\partial_{j}\left(a_{j k} \partial_{k} u+a_{j} u\right)+c_{j} \partial_{j} u+a_{0} u
$$

and

$$
\mathcal{B} u:=\chi\left(\partial_{\nu} u+b \gamma u\right)+(1-\chi) \gamma u
$$

Suppose that

$$
s<\sigma<2-n / q^{\prime}
$$

and define $A(t)$ and $B(t)$ as above. (Note that

$$
W_{q, \mathcal{B}_{\pi}(t)}^{r}=W_{q, \mathcal{B}}^{r}, \quad-2+1 / q<r<1+1 / q \text { with } r \notin \mathbb{Z}+1 / q,
$$

so that these operators are well-defined.) Then (14.7) holds in this case also. Moreover,

$$
\begin{aligned}
& \langle v,(A(t)+B(t)) u\rangle_{W_{q, \mathcal{B}}^{s-2}} \\
& \quad=\left\langle\partial_{j} v, a_{j k} \partial_{k} u+a_{j} u\right\rangle+\left\langle v, c_{j} \partial_{j} u+a_{0} u\right\rangle+\langle\gamma v, \chi b \gamma u\rangle_{\Gamma}
\end{aligned}
$$

for $(v, u) \in W_{q^{\prime}, \mathcal{B}^{\sharp}}^{2-s} \times W_{q, \mathcal{B}}^{s}$. Of course, $A(t)+B(t)$ is again called $W_{q, \mathcal{B}}^{s-2}$ realization of $(\mathcal{A}(t), \mathcal{B}(t))$.

\section{MAXIMAL REgULARITY}

The following theorem gives a maximal regularity result if the lower order terms are bounded in time.

Theorem 14.2. Let assumptions (14.1) and (14.2) be satisfied. Also suppose that either (14.3) and (14.6) or (14.8) and (14.11) hold. If $\boldsymbol{\alpha} \in L_{\infty}((0, T), \mathbb{L})$, then

$$
A+B \in \mathcal{M R}_{p}\left([0, T],\left(W_{q, \mathcal{B}}^{s}, W_{q, \mathcal{B}}^{s-2}\right)\right)
$$

and

$$
(A+B)^{\bullet} \in \mathcal{M R}_{p^{\prime}}\left([0, T],\left(W_{q^{\prime}, \mathcal{B}^{\sharp}}^{2-s}, W_{q^{\prime}, \mathcal{B}^{\sharp}}^{-s}\right)\right)
$$

for $1<p<\infty$. 
Proof. (i) It is not difficult to see that $A \in C\left([0, T], \mathcal{L}\left(W_{q, \mathcal{B}}^{s}, W_{q, \mathcal{B}}^{s-2}\right)\right)$. From (13.7) we infer that $B \in L_{\infty}\left((0, T), \mathcal{L}\left(W_{q, \mathcal{B}}^{s}, W_{q, \mathcal{B}}^{\sigma-2}\right)\right)$. Thus the first assertion follows from Theorems 3.2 and 12.2 , because

$$
W_{q, \mathcal{B}}^{\sigma-2} \hookrightarrow\left(W_{q, \mathcal{B}}^{s-2}, W_{q, \mathcal{B}}^{s}\right)_{\vartheta, \infty}
$$

for $0<2 \vartheta<\sigma-s$ by [4, Theorem V.1.5.3].

One verifies that $(A+B)^{\bullet}=A^{\bullet}+B^{\bullet}$, where $B^{\bullet}(t)=C(T-t)$. Hence (13.8) implies

$$
B^{\bullet} \in L_{\infty}\left((0, T), \mathcal{L}\left(W_{q^{\prime}, \mathcal{B}^{\sharp}}^{2-\sigma}, W_{q^{\prime}, \mathcal{B}^{\sharp}}^{-s}\right)\right) .
$$

The almost reiteration theorem [4, Theorem V.1.5.3] guarantees that

$$
\left(W_{q^{\prime}, \mathcal{B}^{\sharp}}^{2-s}, W_{q^{\prime}, \mathcal{B} \sharp}^{-s}\right)_{\theta, \infty} \hookrightarrow W_{q^{\prime}, \mathcal{B}^{\sharp}}^{2-\sigma},
$$

provided $2>2 \theta>2+s-\sigma$. Thus the second assertion follows also from Theorems 3.2 and 12.2 .

\section{Measures and Singular Distributions}

For $\mu \in \mathcal{M}=\mathcal{M}\left(\Omega, \mathbb{R}^{N}\right)$ we denote by $\mu^{0}$ the completion of the Borel measure

$$
\mathfrak{B}_{\bar{\Omega}} \rightarrow \mathbb{R}^{N}, \quad B \mapsto \mu(B \cap \Omega),
$$

where $\mathfrak{B}_{\bar{\Omega}}$ is the Borel $\sigma$-algebra of $\bar{\Omega}$. Then $\mu^{0} \in \mathcal{M}(\bar{\Omega})$ and the map

$$
\mathcal{M} \rightarrow \mathcal{M}(\bar{\Omega}), \quad \mu \mapsto \mu^{0}
$$

is a linear isometry. We call $\mu^{0}$ trivial extension of $\mu$ and put

$$
\int_{\Omega} \varphi d \mu:=\int_{\bar{\Omega}} \varphi d \mu^{0}, \quad \varphi \in C_{0}(\bar{\Omega}) .
$$

Henceforth, we do not distinguish between $\mu \in \mathcal{M}$ and $\mu^{0} \in \mathcal{M}(\bar{\Omega})$, that is, we identify $\mu$ with its trivial extension.

Clearly, $C_{0}^{1}(\bar{\Omega})$ is the space of all $\varphi \in C_{0}(\bar{\Omega})$ such that $\partial_{j} \varphi$ belongs to $C_{0}(\bar{\Omega})$ for $1 \leq j \leq n$. Thus $C_{0}^{1}(\bar{\Omega})=C^{1}(\bar{\Omega})$ if $\Omega$ is bounded.

Given $\nu_{0} \in \mathcal{M}$ and $\vec{\nu}:=\left(\nu_{1}, \ldots, \nu_{n}\right) \in \mathcal{M}(\bar{\Omega})^{n}$, we set

$$
\langle\mu, \varphi\rangle_{C^{1}}:=\int_{\Omega} \varphi d \nu_{0}-\sum_{j=1}^{n} \int_{\bar{\Omega}} \partial_{j} \varphi d \nu_{j}
$$

for $\varphi \in C_{0}^{1}(\bar{\Omega}) \cap C_{0}=\left\{\psi \in C_{0}^{1}(\bar{\Omega}) ; \gamma \psi=0\right\}$. Then $\mu$ is a continuous linear form on $C_{0}^{1}(\bar{\Omega}) \cap C_{0}$, denoted by $\nu_{0}+\nabla \cdot \vec{\nu}$. We write $\mathcal{M}^{-1}$ for the set of all $\mu \in\left(C_{0}^{1}(\bar{\Omega}) \cap C_{0}\right)^{\prime}$ for which there exists $\left(\nu_{0}, \vec{\nu}\right) \in \mathcal{M} \times \mathcal{M}(\bar{\Omega})^{n}$ satisfying

$$
\mu=\nu_{0}+\nabla \cdot \vec{\nu}
$$

It is a Banach space with the norm

$$
\|\mu\|_{\mathcal{M}^{-1}}:=\inf \left(\left\|\nu_{0}\right\|_{\mathcal{M}}+\|\vec{\nu}\|_{\mathcal{M}(\bar{\Omega})^{n}}\right)
$$

the infimum being taken over all representations (15.2).

Proposition 15.1. $\mathcal{M}^{-1} \doteq\left(C_{0}^{1}(\bar{\Omega}) \cap C_{0}\right)^{\prime}$. 
Proof. It is obvious that

$$
\mathcal{M}^{-1} \hookrightarrow\left(C_{0}^{1}(\bar{\Omega}) \cap C_{0}\right)^{\prime} .
$$

The map

$$
S: C_{0}^{1}(\bar{\Omega}) \cap C_{0} \rightarrow C_{0} \times C_{0}(\bar{\Omega})^{n}, \quad \varphi \mapsto\left(\varphi_{0},-\left(\partial_{1} \varphi, \ldots, \partial_{n} \varphi\right)\right)
$$

is a linear isometry, if the image space is given the $\ell_{\infty}$ product norm. Thus $E:=S\left(C_{0}^{1}(\bar{\Omega}) \cap C_{0}\right)$ is a closed linear subspace of $C_{0} \times C_{0}(\bar{\Omega})^{n}$, and $S$ is an isomorphism from $C_{0}^{1}(\bar{\Omega}) \cap C_{0}$ onto $E$. The dual of $C_{0} \times C_{0}(\bar{\Omega})^{n}$ equals $\mathcal{M} \times \mathcal{M}(\bar{\Omega})^{n}$, where the latter space carries the $\ell_{1}$ product norm. Thus $\mathrm{N}:=\left(\nu_{0}, \vec{\nu}\right) \in \mathcal{M} \times \mathcal{M}(\bar{\Omega})^{n}$ defines a continuous linear form, namely $\mathrm{N} \circ S$, on $C_{0}^{1}(\bar{\Omega}) \cap C_{0}$. Note that $\langle\mathrm{N} \circ S, \varphi\rangle$ equals the right-hand side of (15.1) for $\varphi \in C_{0}^{1}(\bar{\Omega}) \cap C_{0}$, that is,

$$
S^{\prime}(\mathrm{N} \mid E)=\nu_{0}+\nabla \cdot \vec{\nu} \in \mathcal{M}^{-1}
$$

From this and (15.3) it follows that $\mathcal{M}^{-1}$ and $\left(C_{0}^{1}(\bar{\Omega}) \cap C_{0}\right)^{\prime}$ coincide as vector spaces. Now the assertion follows from (15.3) and Banach's homomorphism theorem.

We also set

$$
\mathcal{M}^{-1}(\Gamma):=C^{1}(\Gamma)^{\prime}
$$

Then we define closed linear subspaces $\mathcal{M}_{1}^{-1}(\Gamma)$ of $\mathcal{M}^{-1}(\Gamma)$ and $\mathcal{M}_{0}(\Gamma)$ of $\mathcal{M}(\Gamma)$ by

$$
\mathcal{M}_{1}^{-1}(\Gamma):=\left\{\mu \in \mathcal{M}^{-1}(\Gamma) ;(1-\chi) \mu=0\right\}
$$

and

$$
\mathcal{M}_{0}(\Gamma):=\{\mu \in \mathcal{M}(\Gamma) ; \chi \mu=0\},
$$

respectively. Hence the direct sum

$$
\mathcal{M}_{\chi}^{-1}(\Gamma):=\mathcal{M}_{0}(\Gamma) \oplus \mathcal{M}_{1}^{-1}(\Gamma)
$$

is a well-defined Banach space. Note that, given $\mu \in \mathcal{M}_{\chi}^{-1}(\Gamma)$, its $r$-th component is a singular distribution in $C^{1}\left(\Gamma_{1}^{r}, \mathbb{R}\right)^{\prime}$ on $\Gamma_{1}^{r}$, and a real valued Radon measure on $\Gamma_{0}^{r}$. Also note that the natural injection

$$
\mathcal{M}(\Gamma) \rightarrow \mathcal{M}_{\chi}^{-1}(\Gamma), \quad \mu \mapsto(1-\chi) \mu+\chi \mu
$$

is continuous. We express this by writing

$$
\mathcal{M}(\Gamma) \hookrightarrow \mathcal{M}_{\chi}^{-1}(\Gamma)
$$

We also put

$$
\mathcal{M}_{\chi}(\Gamma):=\{\mu \in \mathcal{M}(\Gamma) ;(1-\chi) \mu=0\} .
$$

Thus $\mathcal{M}_{\chi}(\Gamma)$ is the closed linear subspace of $\mathcal{M}(\Gamma)$ consisting of all $\mu$ whose $r$-th component vanishes on $\Gamma_{0}^{r}$. 
16. Parabolic equations With measures

Throughout this section we presuppose hypotheses (14.1) and (14.2). We also suppose that either

- $\left.\begin{array}{l}\overrightarrow{\mathcal{M}}:=\mathcal{M}^{-1} \times \mathcal{M}_{\chi}^{-1}(\Gamma) \\ \text { - }\end{array}\right\}$

or

- hypothesis (14.8) holds and

- $\overrightarrow{\mathcal{M}}:=\mathcal{M} \times \mathcal{M}_{\chi}(\Gamma)$.

We define $(\mathcal{A}, \mathcal{B})$ by $(14.4),(14.5)$ and $(14.9),(14.10)$, respectively. We also set

$$
\mathcal{A}^{\sharp} v:=-\partial_{k}\left(a_{j k}^{\top} \partial_{j} v\right)+a_{j}^{\top} \partial_{j} v+a^{\top} v
$$

if assumption (14.3) holds, and

$$
\mathfrak{a}(v, w):=\left\langle\partial_{j} v, a_{j k} \partial_{k} w+a_{j} w\right\rangle+\left\langle v, c_{j} \partial_{j} w+a w\right\rangle+\langle\gamma v, \chi b \gamma w\rangle_{\Gamma}
$$

otherwise. Moreover, $\partial_{\boldsymbol{\nu}^{\sharp}}$ is defined by replacing $a_{j k}$ in (12.2) by $a_{j k}^{\top}$.

Suppose that

$$
\vec{\mu}=\left(\mu_{\Omega}, \mu_{\Gamma}\right) \in \mathcal{M}([0, T), \overrightarrow{\mathcal{M}})
$$

and consider the (formal) parabolic system

$$
\begin{aligned}
\partial_{t} u+\mathcal{A} u=\mu_{\Omega} & \text { in } \Omega \times[0, T), \\
\mathcal{B} u=\mu_{\Gamma} & \text { on } \Gamma \times[0, T) .
\end{aligned}
$$

By an $L_{r}\left(W_{q}^{s}\right)$ solution of (16.1) we mean a function $u \in L_{r}\left([0, T), W_{q, \mathcal{B}}^{s}\right)$ satisfying

$$
\begin{aligned}
\int_{0}^{T}\langle & \left.-\dot{\varphi}+\mathcal{A}^{\sharp} \varphi, u\right\rangle d t \\
& =\int_{[0, T)} \varphi d \mu_{\Omega}-\int_{[0, T)}(1-\chi) \partial_{\boldsymbol{\nu} \sharp} \varphi d \mu_{\Gamma}+\int_{[0, T)} \chi \gamma \varphi d \mu_{\Gamma}
\end{aligned}
$$

for all $\varphi \in \mathcal{D}([0, T), \mathcal{D}(\bar{\Omega}))$ satisfying $(1-\chi) \gamma \varphi=0$ and $\chi \partial_{\boldsymbol{\nu}} \varphi=0$, provided hypothesis (14.3) is satisfied. If assumption (14.8) holds then (16.2) is to be replaced by:

$$
\int_{0}^{T}\{\langle-\dot{\varphi}, u\rangle+\mathfrak{a}(\varphi, u)\} d t=\int_{[0, T)} \varphi d \mu_{\Omega}+\int_{[0, T)} \chi \gamma \varphi d \mu_{\Gamma}
$$

for all $\varphi \in \mathcal{D}([0, T), \mathcal{D}(\bar{\Omega}))$ satisfying $(1-\chi) \gamma \varphi=0$

Theorem 16.1. Suppose that $\boldsymbol{\alpha} \in L_{\rho}((0, T), \mathbb{L})$, where

$$
0 \leq 2 / \rho \leq 2-2 / r<1-n / q^{\prime}-s
$$

if (14.3) is satisfied, and

$$
0 \leq 2 / \rho \leq 2-2 / r<2-n / q^{\prime}-s
$$

otherwise. Then:

(i) (Existence and uniqueness) Problem (16.1) possesses for each $\vec{\mu}$ belonging to $\mathcal{M}([0, T), \overrightarrow{\mathcal{M}})$ a unique $L_{r}\left(W_{q}^{s}\right)$ solution. 
(ii) (Continuous dependence on $\vec{\mu}$ ) The solution map $\vec{\mu} \rightarrow u$ is linear and continuous from $\mathcal{M}([0, T), \overrightarrow{\mathcal{M}})$ into $L_{r}\left((0, T), W_{q, \mathcal{B}}^{s}\right)$. It is also continuous if these spaces are given the weak star and weak topology, respectively.

(iii) (Continuous dependence on the coefficients) The solution is continuous as a map from

$$
\begin{aligned}
& C\left([0, T], \mathcal{E} \ell \ell^{1}(\Omega)\right) \times L_{\rho}((0, T), \mathbb{L}) \times \mathcal{M}([0, T), \overrightarrow{\mathcal{M}}) \\
& \text { into } L_{r}\left((0, T), W_{q, \mathcal{B}}^{s}\right) .
\end{aligned}
$$

(iv) (Compactness) If $\Omega$ is bounded then the solution map is compact on bounded sets.

Proof. Fix $\sigma \in\left(s, 1-n / q^{\prime}\right)$ if (14.3) is satisfied, and $\sigma \in\left(s, 2-n / q^{\prime}\right)$ otherwise, and $p$ such that $r<p<2 /(2+s-\sigma)$. This is possible because of (16.3) and (16.4), respectively.

Set $2 \alpha:=s$ and $2 \beta:=\sigma$. Then Theorems 12.1(iv) and 14.2 (with $B=0$ ) imply that

$$
A \in \mathcal{M R}_{p}\left([0, T],\left(F_{\alpha}, F_{\alpha-1}\right)\right) \quad \text { and } \quad A^{\bullet} \in \mathcal{M R}_{p^{\prime}}\left([0, T],\left(F_{1-\alpha}^{\sharp}, F_{-\alpha}^{\sharp}\right)\right) .
$$

From (13.7) and Hölder's inequality we infer that $B$ satisfies (10.3). Set

$$
\mu:=\mu_{\Omega}-\left(\partial_{\boldsymbol{\nu}^{\sharp}}\right)^{\prime}(1-\chi) \mu_{\Gamma}+\gamma^{\prime} \chi \mu_{\Gamma}
$$

if (14.3) is satisfied, and $\mu:=\mu_{\Omega}+\gamma^{\prime} \chi \mu_{\Gamma}$ otherwise. Here

$$
\left(\partial_{\boldsymbol{\nu}^{\sharp}}\right)^{\prime} \in \mathcal{L}\left(W_{q}^{\sigma-1 / q}(\Gamma), W_{q, \mathcal{B}}^{\sigma-2}\right)
$$

is the dual of $\partial_{\boldsymbol{\nu}^{\sharp}} \in \mathcal{L}\left(W_{q^{\prime}, \mathcal{B}^{\sharp}}^{2-\sigma}, W_{q^{\prime}}^{1 / q-\sigma}(\Gamma)\right)$, and

$$
\gamma^{\prime} \in \mathcal{L}\left(W_{q}^{\sigma-1-1 / q}(\Gamma), W_{q, \mathcal{B}}^{\sigma-2}\right)
$$

is the dual of $\gamma \in \mathcal{L}\left(W_{q^{\prime}, \mathcal{B}^{\sharp}}^{2-\sigma}, W_{q^{\prime}}^{1+1 / q-\sigma}(\Gamma)\right)$. It follows from [10, Lemma 4.1] that

$$
\mathcal{M}([0, T), \overrightarrow{\mathcal{M}}) \rightarrow \mathcal{M}\left([0, T), W_{q, \mathcal{B}}^{\sigma-2}\right), \quad \vec{\mu} \mapsto \mu
$$

is a well-defined bounded linear map. Now the assertion is an easy consequence of Theorem 10.1.

\section{A WEAK MAXIMUM PRINCIPLE}

In this last section we prove a positivity result for parabolic systems, given the preceding low regularity assumptions on the coefficients. It is a version of a weak maximum principle and extends a corresponding result for elliptic systems in [7].

We impose the conditions:

- Assumptions (14.1) and either (14.3) or (14.8) are satisfied;

- $a_{j k}, a_{j}$, and $c_{j}$ are diagonal matrices for $1 \leq j, k \leq n$. $\}$

The matrices $a_{0}$ and $b$ are not supposed to be diagonal but to satisfy the following "cooperativity" hypotheses:

$$
a^{\alpha \beta}, b^{\alpha \beta} \leq 0, \quad 1 \leq \alpha, \beta \leq N, \quad \alpha \neq \beta .
$$

The following weak maximum principle is deduced from Theorem 9.1. 
Theorem 17.1. Let assumptions (17.1) and (17.2), as well as (16.3), respectively (16.4), be satisfied. If $\vec{\mu} \in \mathcal{M}([0, T], \overrightarrow{\mathcal{M}})$ is positive then the unique $L_{r}\left(W_{q}^{s}\right)$ solution of $(16.1)$ is also positive.

Proof. Set $\mathbb{M}:=\left(\boldsymbol{B} \boldsymbol{U} \boldsymbol{C}_{\Gamma}^{1}\right)^{n+1}$ if (14.3) holds, and

$$
\mathbb{M}:=\left(B \boldsymbol{U} \boldsymbol{C}_{\Gamma}^{1}\right)^{2 n+1} \times \boldsymbol{C}^{1}(\Gamma)
$$

otherwise. Note that (13.1) implies that $\mathbb{M}$ is dense in $\mathbb{L}$.

Suppose that

$$
\left(\left[a_{j k}\right], \boldsymbol{\alpha}\right) \in C^{1}\left([0, T], \mathcal{E} \ell \ell^{1}(\Omega) \times \mathbb{M}\right) .
$$

Define $(\widetilde{\mathcal{A}}, \widetilde{\mathcal{B}})$ by $\widetilde{\mathcal{A}}:=\mathcal{A}$ and $\widetilde{\mathcal{B}}:=\mathcal{B}+\chi\left(\gamma a_{j}\right) \nu^{j} \gamma$. Let $\left(\widetilde{\mathcal{A}}^{\sharp}, \widetilde{\mathcal{B}}^{\sharp}\right)$ be the boundary value problem formally dual to $(\widetilde{\mathcal{A}}, \widetilde{\mathcal{B}})$ (see $[3$, Section 7$]$ ). Note that

$$
\widetilde{\mathcal{B}}=\mathcal{B}, \quad \widetilde{\mathcal{B}}^{\sharp}=\mathcal{B}^{\sharp}:=\chi\left(\partial_{\boldsymbol{\nu}^{\sharp}}+b^{\top} \gamma\right)+(1-\chi) \gamma
$$

because $a_{j}(t)\left|\Gamma=c_{j}(t)\right| \Gamma=0$. Denote by $\widetilde{A}_{0}(t)$ the $L_{q}$ realization of $(\widetilde{\mathcal{A}}(t), \widetilde{\mathcal{B}}(t))$. Then $\widetilde{A}_{0}(t) \in \mathcal{H}\left(W_{q, \widetilde{\mathcal{B}}(t)}^{2}, L_{q}\right)$, so that $\left[\widetilde{F}_{\beta}(t) ;|\beta| \leq 1\right]$, the interpolation extrapolation scale generated by $\left(L_{q}, \widetilde{A}_{0}(t)\right)$ and the interpolation functors (12.4), is well-defined. From (17.4) and [3, Theorem 7.1] we infer that

$$
A(t)+B(t)=\widetilde{A}_{s-2}(t), \quad 0 \leq t \leq T,
$$

where $\widetilde{A}_{s-2}(t)$ is the $W_{q, \mathcal{B}}^{s-2}$ realization of $(\widetilde{\mathcal{A}}(t), \widetilde{\mathcal{B}}(t))$. From [4, Theorem V.2.1.3] we know that $\widetilde{A}_{0}(t)$ is resolvent positive. Hence $A(t)+B(t)$ belongs to $\mathcal{H}^{+}\left(E_{1}, E_{0}\right)$, where $\left(E_{1}, E_{0}\right):=\left(W_{q, \mathcal{B}}^{s}, W_{q, \mathcal{B}}^{s-2}\right)$. Consequently,

$$
(A(t)+B(t))^{\bullet} \in \mathcal{H}^{+}\left(E_{1}^{\bullet}, E_{0}^{\bullet}\right) .
$$

From $(A+B)^{\bullet}=A^{\bullet}+B^{\bullet}$ and $B^{\bullet}(t)=C(T-t)$ and (17.3) we infer, because of (13.8), that

$$
(A+B)^{\bullet} \in C^{1}\left([0, T], \mathcal{H}^{+}\left(E_{1}^{\bullet}, E_{0}^{\bullet}\right)\right) .
$$

Now we infer from Theorems 14.2 and 9.1 that $\left(K_{A+B}\right)^{\bullet}$ is positive. Hence its dual, $\left[\left(K_{A+B}\right)^{\bullet}\right]^{\prime}$, is positive as well. This proves the assertion if (17.3) is true.

Since $C^{1}\left([0, T], \mathcal{E} \ell \ell^{1}(\Omega) \times \mathbb{M}\right)$ is dense in

$$
C^{1}\left([0, T], \mathcal{E} \ell \ell^{1}(\Omega) \times L_{\rho}((0, T) \times \mathbb{L})\right)
$$

the assertion in the general case follows now by an obvious approximation argument from Theorem 16.1(iii).

\section{REFERENCES}

[1] H. Amann. Existence and regularity for semilinear parabolic evolution equations. Ann. Scuola Norm. Sup. Pisa, Ser. IV, 11 (1984), 593-676.

[2] H. Amann. Dynamic theory of quasilinear parabolic equations. II. Reactiondiffusion systems. Differential Integral Equations, 3(1) (1990), 13-75.

[3] H. Amann. Nonhomogeneous linear and quasilinear elliptic and parabolic boundary value problems. In Function spaces, differential operators and nonlinear analysis (Friedrichroda, 1992), volume 133 of Teubner-Texte Math., pages 9-126. Teubner, Stuttgart, 1993. 
[4] H. Amann. Linear and quasilinear parabolic problems. Vol. I, volume 89 of Monographs in Mathematics. Birkhäuser Boston Inc., Boston, MA, 1995. Abstract linear theory.

[5] H. Amann. Linear parabolic problems involving measures. Rev. R. Acad. Cien. Serie A. Mat. (RACSAM), 95 (2001), 85-119.

[6] H. Amann. Maximal regularity for nonautonomous evolution equations, 2003. Preprint.

[7] H. Amann. Maximum principles and principal eigenvalues, 2003. To appear.

[8] H. Amann, M. Hieber, G. Simonett. Bounded $H_{\infty}$-calculus for elliptic operators. Diff. Int. Equ., 7 (1994), 613-653.

[9] H. Amann, P. Quittner. Control problems governed by semilinear parabolic equations with non-monotone nonlinearities and low regularity data, 2003. Preprint.

[10] H. Amann, P. Quittner. Semilinear parabolic equations involving measures and low-regularity data. Trans. Amer. Math. Soc., (2003). To appear.

[11] J. Bergh, J. Löfström. Interpolation Spaces. An Introduction. Springer Verlag, Berlin, 1976.

[12] R. Denk, G. Dore, M. Hieber, J. Prüss, A. Venni. New thoughts on old results of R.T. Seeley, 2003. Preprint.

[13] R. Denk, M. Hieber, J. Prüss. $\mathcal{R}$-boundedness, Fourier multipliers and problems of elliptic and parabolic type. Memoires Amer. Math. Soc., (2003). To appear.

[14] G. Dore. $L^{p}$ regularity for abstract differential equations. In H. Komatsu, editor, Functional Analysis and Related Topics, 1991, pages 25-38, Berlin, 1993. Proceedings Kyoto 1991, Lecture Notes in Math. \#1540, Springer Verlag.

[15] P.E. Sobolevskii. Coerciveness inequalities for abstract parabolic equations. Soviet Math. (Doklady), 5 (1964), 894-897.

[16] H. Triebel. Interpolation Theory, Function Spaces, Differential Operators. North Holland, Amsterdam, 1978.

Institut Für Mathematik, Universität Zürich, Winterthurerstr. 190, CH8057 ZÜRICH, SWITZERLAND

E-mail address: amann@math.unizh.ch 